\title{
Le chemin vers la révélation : lumière et nuit dans le proème de Parménide
}

\section{Oliver Primavesi}

\section{(2) OpenEdition}

1 Journals

\section{Édition électronique}

URL : https://journals.openedition.org/philosant/900

DOI : 10.4000/philosant.900

ISSN : 2648-2789

Éditeur

Éditions Vrin

\section{Édition imprimée}

Date de publication : 1 novembre 2013

Pagination : $37-81$

ISBN : 978-2-7574-0591-8

ISSN : 1634-4561

\section{Référence électronique}

Oliver Primavesi, « Le chemin vers la révélation : lumière et nuit dans le proème de Parménide », Philosophie antique [En ligne], 13 | 2013, mis en ligne le 01 novembre 2018, consulté le 03 décembre 2022. URL : http://journals.openedition.org/philosant/900 ; DOI : https://doi.org/10.4000/philosant. 900

\section{(c) (i) ()}

Creative Commons - Attribution - Pas d'Utilisation Commerciale - Pas de Modification 4.0 International - CC BY-NC-ND 4.0

https://creativecommons.org/licenses/by-nc-nd/4.0/ 


\title{
LE CHEMIN VERS LA RÉVÉLATION : LUMIÈRE ET NUIT DANS LE PROÈME DE PARMÉNIDE*
} Oliver PRIMAVESI

Ludwig-Maximilians-Universität, Munich

\begin{abstract}
RÉSUMÉ. Cet article propose une interprétation de la relation entre l'aletheia et la doxa dans le poème de Parménide sur la base d'une analyse du voyage relaté dans le proème. À partir d'un examen précis du texte parménidien, il établit que l'hypothèse selon laquelle la citadelle de la nuit est la destination finale du voyage rend bien mieux compte de celui-ci que l'hypothèse longtemps admise selon laquelle il s'agirait de la lumière. Cette lecture du proème permet non seulement d'établir un certain nombre de parallèles avec d'autres œuvres poétiques qui décrivent le trajet du Soleil, mais surtout de mettre au jour une analogie entre le récit de voyage et la partition en deux du poème parménidien : notre monde, où alternent le jour et la nuit, représente le monde de la doxa, où être et non-être sont mêlés et qui est gouverné par deux principes correspondants, le feu et la nuit, tandis que l'unicité de la déesse de la Nuit dans l'au-delà renvoie à celle de l'être. De plus, en distinguant deux étapes dans le voyage du narrateur, celle où il atteint de lui-même le chemin de la nuit et du jour et celle où les filles d'Hélios le guident sur ce chemin, on peut expliquer l'existence même d'un discours sur les opinions des mortels : de même que le narrateur a besoin d'abord d'aller de notre monde quotidien jusqu'au chemin de la nuit et du jour pour avoir ensuite accès à la connaissance divine, de même il faut expliquer le monde de l'opinion en le faisant remonter à deux principes fondamentaux pour montrer aux mortels le chemin vers l'aletheia. L'article conclut en expliquant, à partir des fragments B14 et B15, pourquoi Parménide a recours à la déesse de la Nuit pour faire cette révélation : ce choix repose sur une critique de la lumière du soleil comme condition de la vision trompeuse.
\end{abstract}

SUMMARY. This article develops an interpretation of the relation between aletheia and doxa in Parmenides' poem on the basis of an analysis of the journey narrated in its proem. The hypothesis that the destination of the journey is the house of night, and

. L'auteur remercie chaleureusement Jaap Mansfeld pour sa lecture de la version allemande de ce texte (Primavesi 2011) ainsi que Mathilde Bremond pour la traduction française.

Philosophie antique, $\mathrm{n}^{\circ} 13$ (2013), 37-81 
not that of light, as was assumed for a long time, is vindicated on the basis of a detailed analysis of the text. This reading of the proem not only allows us to draw a number of parallels with other poetical works which describe the Sun's trajectory, but also and above all to establish an analogy between the narrative of the journey and the division of Parmenides' poem into two parts: our world, where day and night alternate, represents the world of doxa, in which being and non-being are mixed and which is governed by two corresponding principles, fire and night; while the unicity of the night-goddess, beyond our world, refers to being's unicity. Moreover, by distinguishing two moments in the journey of the narrator, one in which he reaches the road of night and day by himself and the other in which Helios' daughters escort him on the road, the very existence of a development dedicated to the opinions of mortals can be explained: just as the narrator must first go from our daily world to the road of night and day in order thereafter to access divine knowledge, in the same way the world of opinion must be explained by reference to two fundamental principles in order to show to the mortals the road towards aletheia. The article concludes by explaining, on the basis of fr. B14 and B15, why Parmenides makes use of the goddess of Night for this revelation: this choice relies on a critique of the sun's light as being the condition of deceiving vision. 


\section{Introduction}

Un des thèmes classiques de l'histoire de la philosophie est la question de la relation entre les deux parties du poème didactique de Parménide: pourquoi la déesse, dans sa révélation au narrateur, présente-t-elle d'abord dans la première partie la vérité (aletheia) qu'elle tire d'un raisonnement logique, pour ensuite enchaîner avec une deuxième partie dans laquelle elle traite en détail des simples opinions (doxai) des mortels sur le monde, opinions qui s'appuient sur la perception sensible, et ce alors que le commun des mortels ignore jour après jour étourdiment l'alternative stricte révélée dans la première partie entre la voie praticable de l'« est » et celle impraticable du «n'est pas »?

On cherche depuis longtemps une clef possible pour résoudre ce problème dans le récit de voyage mythique, intégralement conservé par Sextus Empiricus ${ }^{1}$, qui est placé comme proème devant les deux parties du poème parménidien. Dans les vingt-trois premiers vers, le narrateur raconte comment il parvient avec son attelage d'abord à un «chemin du dieu », puis est conduit sur ce chemin par les filles du dieu Soleil jusqu'à une déesse qui le reçoit avec bienveillance et lui offre une révélation : cette révélation constitue alors l'essentiel du poème.

Cependant, malgré d'intenses débats, aucun consensus ne s'est jusqu'ici formé sur la destination du voyage entrepris par le narrateur, c'est-à-dire sur la localisation de l'habitation de la déesse où arrive le voyageur, et encore moins sur l'identité de la déesse elle-même ou même sur le trajet précis du voyage. Hermann Diels, dans sa fondamentale édition commentée de 1897, était d'avis que le proème de Parménide décrit un chemin du narrateur vers la lumière divine de la connaissance ; il a ainsi fondé l'opinio communis de la première moitié du $\mathrm{XX}^{\mathrm{e}}$ siècle. Cependant cette interprétation, que nous appellerons par la suite « hypothèse de la lumière » et qui s'est développée de deux manières, doit faire face à des incohérences considérables dans le

1. Sextus Empiricus, Adversus Mathematicos, 7, 111 (texte continu) et 112-114 (texte avec paraphrase) $=$ DK $28 \mathrm{~B} 1$. 
texte, ce que les grands défenseurs des deux variantes, Walther Kranz (1916) et Hermann Fränkel (1930), ont bien reconnu. Kranz, du fait de la prétendue absurdité du récit, nie complètement l'originalité poétique de Parménide - jugement douteux, que Kranz cherche donc à adoucir aussi bien en louant encore plus son originalité conceptuelle qu'en affirmant ad hoc que la qualité poétique n'est pas un critère convenable pour juger Parménide ${ }^{2}$ :

Ce voyage poétique n'a pas émergé librement de l'âme imaginative d'un poète comme un ensemble organique, mais ne laisse pas de montrer les éléments d'une construction rationnelle, est rempli d'images conventionnelles et bâti de mots traditionnels. Comment pourrait-il en être autrement ? Le fondateur de la dialectique, qui accomplit le plus grand acte philosophique de la pensée prékantienne, devrait donc avoir été poète ?

Fränkel s'est même vu contraint à aller encore plus loin : il oppose un refus à toute tentative « de vouloir déterminer le trajet en question ainsi que son emplacement $\gg^{3}$ :

De telles tentatives ont souvent été lancées; elles n'ont produit aucun résultat qui reste encore plausible quand on le met directement à côté du texte. Les mots de Parménide montrent au contraire qu'il n'y a pas de trajet localisable .

On peut néanmoins se demander si ce constat décevant est véritablement à imputer au texte de Parménide, ou s'il ne montre pas tout simplement que l'hypothèse de la lumière, admise comme axiome par Kranz et Fränkel à la suite de Diels, en fait ne rend pas justice à ce texte.

Face à ce grave dilemme, la contre-hypothèse, exposée en premier par Morrison (1955), mérite attention : selon lui, le voyage du narrateur ne se fait pas vers la lumière, mais vers la citadelle de la Nuit (B 1, 9: $\delta \dot{\omega} \mu \alpha \tau \alpha$ Nukтós), située dans l'au-delà. Nous appellerons par la suite cette interprétation « hypothèse de la nuit ».

2. Kranz 1916, p. 1163 : «Diese Dichterreise ist nicht aus der bilderreichen Seele eines Dichters als lebendiges und Ganzes frei emporgestiegen, sondern ist mit Elementen konstruierender Verstandestätigkeit durchsetzt, mit überkommenen Bildern gefüllt, aus traditionellen Worten geschichtet. Wie könnte es auch anders sein: Der Begründer der Dialektik, dessen philosophische Tat die größte der vorkantischen Philosophie ist, sollte ein Poet gewesen sein? 》

3. Fränkel 1930, p. 157 (= 161): «den gemeinten Fabrtweg nebst seinen Stationen ermitteln zu wollen. 》

4.Ibidem: «Solche Versuche sind mehrfach unternommen worden; sie haben kein Ergebnis gezeitigt, das auch dann einleuchtend bliebe, wenn man es unmittelbar neben den Text stellt. Die Worte des Parmenides zeigen vielmehr, daß es einen lokalisierbaren Fahrtweg nicht gibt.» 
L'innovation de Morrison n'a reçu aucune attention de la part de Deichgräber (1958), qui présuppose au contraire l'hypothèse de la lumière comme si elle allait de soi. Cependant, en 1964, dans une discussion approfondie de l'état de la recherche sur la question, Jaap Mansfeld a miné des suppositions essentielles de l'hypothèse de la lumière et tiré profit dans sa propre interprétation du voyage de la plus grande cohérence structurelle de l'hypothèse de la nuit, en faisant référence à Morrison 5 . Walter Burkert consolide en 1969 les principaux aspects de l'interprétation de Morrison grâce à une argumentation approfondie concernant la grammaire et l'histoire des thématiques. Il établit en particulier que le voyage du narrateur parménidien, si l'on accepte la nouvelle lecture du texte proposée par Morrison, n'est pas une descente aux enfers (Katabasis), mais un voyage jusqu'à l'au-delà, à la limite du disque terrestre, comme l'a déjà entrepris l'Ulysse d'Homère - même si celui-ci voulait invoquer les morts (Nekyia) ${ }^{6}$. De fait, on ne trouve chez Parménide, indépendamment de toute controverse interprétative, aucune mention d'une catabase comme celle dont on trouve le modèle le plus expressif chez Virgile dans le sixième livre de l'Énéide. Ainsi, Morrison a fait peser sur son hypothèse de la nuit un fardeau absolument inutile en mélangeant la terminologie des deux types de voyage dans l'au-delà ${ }^{7}$. À partir de là, le titre de l'article de Burkert («le poème de Parménide et la catabase de Pythagore $\left.{ }^{8} »\right)$ a été mal compris par des lecteurs superficiels : de fait, le but de Burkert est de comparer le voyage décrit dans le proème de Parménide avec la légendaire catabase de Pythagore d'un point de vue fonctionnel, c'est-à-dire pour le gain d'une connaissance surhumaine ${ }^{9}$; malgré cet objectif, Burkert maintient naturellement

5. Mansfeld 1964, p. 222-247. Cependant, il s'écarte p. 244-247 de Morrison en ce qu'il met en relation la description du chemin présentée dans l'hypothèse de la nuit non pas, comme celui-ci, avec la topographie mythologique de la Théogonie d'Hésiode, mais d'une part avec la topographie cosmologique de la doxa parménidienne, d'autre part même avec le monde quotidien (voir p. 227-228); sur ce dernier point, sa représentation repose sur la lecture erronée par Mutschmann du manuscrit $\mathrm{N}$ de Sextus (Laur. 85, 19), attestée depuis comme intenable, à savoir qu'aux vers $2-3$ du proème, le chemin du dieu conduirait l'hom-

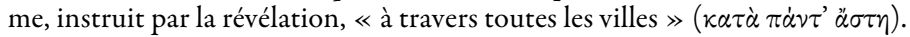

6. Burkert 1969, p. 15 (=13): « il faut cependant plutôt laisser complètement de côté la verticalité, le haut et le bas. L'au-delà n'est dans la représentation archaïque ni en haut ni en bas, mais juste extrêmement éloigné. Ulysse aussi, dans la « Nekyia », ne part ni vers le haut ni vers le bas, mais tout droit vers le lointain. »

7. Morrison 1955, p. $60:$ : Le poète est porté dans son char jusqu'aux confins de la terre, l'emplacement propre à une catabase »; cela se retrouve chez Palmer 2009, p. 53.

8. « Das Proömium des Parmenides und die Katabasis des Pythagoras »

9. Comparer ne veut pas dire assimiler. Au demeurant, la conclusion de l'étude de Burkert montre aussi qu'il voyait parfaitement les différences entre «Myth and Ritual» d'un côté et la philosophie grecque de l'autre (idem, p. $28(=25)$ : «Quoi qu'il s'y cache en 
une différence typologique claire entre le voyage dans l'au-delà selon le modèle de la nekyia et celui selon le modèle de la catabase.

L'hypothèse de la nuit, telle qu'elle est proposée par Morrison et développée par Mansfeld et par Burkert, fut reçue très différemment dans les domaines anglo-saxon et allemand. Dans la recherche anglophone, elle fut établie sous sa forme la plus développée au plus tard en 1973 grâce aux « Notes on Parmenides» dans lesquelles David J. Furley, en introduction aux hommages à Gregory Vlastos, a adopté sans réserve les principaux résultats de Morrison, Mansfeld et Burkert ${ }^{10}$. Ainsi, la recherche anglosaxonne n'a pas négligé l'hypothèse de la nuit: elle fut acceptée par certains ${ }^{11}$, mise en doute par d'autres ${ }^{12}$, mais en tout cas discutée.

En Allemagne, au contraire, l'autorité des travaux d'Hermann Diels, de Walther Kranz et d'Hermann Fränkel, qui se sont ménagé une place dominante dans la recherche allemande sur Parménide antérieure à 1933, a longtemps empêché qu'il soit ne serait-ce que pris acte de la contribution non orthodoxe de Morrison ${ }^{13}$. L'opinio communis y passa longtemps sous silence l'hypothèse de la nuit, même après l'essai de Burkert en 1969. Dans les deux influentes éditions de Parménide faites par Uvo Hölscher (1969) et Ernst Heitsch (1974), le travail de Morrison n'est pas mentionné une seule fois dans la bibliographie, et sa révision lors de la réédition de ces travaux (Hölscher 1986 et Heitsch 1991) n'y change rien ; même l'article de Burkert n'y est pas pris en compte. De plus, Hölscher et Heitsch, comme déjà Fränkel, ne laissent pas voir le moindre doute sur l'hypothèse de la lumière,

matière de rites extérieurs et d'expérience intérieure, la philosophie de Parménide doit

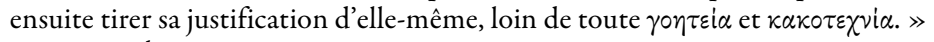

10. Furley 1973, p. 1-5.

11. Par exemple Nussbaum 1979, p. 69 ; Gallop 1984, p. 6-7 ; Sedley 1999, p. 124 et plus récemment Palmer 2009 p. 51-61.

12. Parmi les sceptiques, Owens 1979 donne à son travail le titre trompeur par rapport à la question «Knowledge and "Katabasis" in Parmenides». Curd 1998, p. 19, est agnostique.

13. Pour un lecteur de langue allemande, la variante de l'hypothèse de la lumière proposée par Kranz en 1916 resta d'actualité par la réimpression continuelle de sa réédition des Fragmente der Vorsokratiker (voir Diels/Kranz 1934, p. 228), et Fränkel 1930 a présenté sa version de l'hypothèse, qui s'accorde de plus près avec Diels, dans ses deux ouvrages qui ont connu une large diffusion, Wege und Formen frühgriechischen Denkens (voir Fränkel 1960, p. 157-197) et Dichtung und Philosophie des frühen Griechentums (voir Fränkel 1962, p. 399 : « chaque fois qu'il [Parménide] se plongeait dans ses grandes idées, il se sentait emporté dans un royaume de lumière au-delà du monde terrestre. Dans l'introduction de son poème didactique, il décrit ce processus de ravissement; et comme la langue habituelle ne parvient pas à raconter cet événement inhabituel, il le dépeint avec des images et des symboles. » 
bien qu'ils reconnaissent ouvertement les difficultés qu'elle pose ${ }^{14}$. La même chose vaut pour Hans von Steuben, qui a inséré dans une édition spéciale des fragments de Parménide, publiée en 1981, un essai qui pousse à l'extrême l'hypothèse de la lumière ${ }^{15}$, bien que les suppositions herméneutiques qu'elle nécessite laissent l'auteur lui-même expressément insatisfait $^{16}$. Enfin, Günther (1998) évite certes quelques faiblesses de la lecture traditionnelle dans son interprétation du proème, qui repose sur la philosophie de Heidegger, mais se montre fort peu intéressé à présenter une image topographiquement cohérente du voyage.

Si l'hypothèse de la nuit a d'abord eu peu d'écho dans la recherche germanophone, ce n'était pas le seul obstacle à l'exploitation philosophique du voyage. Mais la discussion de cette hypothèse, là où elle a été menée, s'est développée d'une manière toujours plus stérile pour le problème de la révélation parménidienne. L'hypothèse a d'abord été présentée pour sortir de l'aporie dans laquelle les tenants de l'hypothèse de la lumière, de leur propre aveu, étaient tombés dans leur explication du voyage. Pour établir qu'elle offre véritablement une telle solution, il aurait fallu d'abord, en confrontant systématiquement les deux hypothèses, examiner laquelle atteint le plus haut degré de cohérence dans l'interprétation du récit de voyage, et reconstitue au mieux le rapport entre celui-ci et la révélation. Mais cette tâche a été perdue de vue avant d'avoir été menée à bien.

Au contraire, comme l'hypothèse de la nuit a mis au jour, quand elle a été découverte, un nombre impressionnant de points communs avec des références extérieures, les chercheurs ont été conduits à se concentrer sur les relations possibles entre le proème et des sources externes comme les mythes traditionnels de catabase ou les Lamelles d'or orphiques plutôt que sur le texte du proème et sa relation à la révélation finale. Ainsi, Sassi

14. Hölscher 1969, p. 70-71: « Mais si l'on cherche à déterminer ne serait-ce que les étapes du voyage, on tombe aussitôt dans des difficultés... Chez Parménide, les métaphores ne s'unissent pas pour former le tout d'une intuition réelle qui puisse servir de médiateur entre l'image et le sens. »; Heitsch 1974, p. 134 : « Il semble douteux qu'il ait voulu esquisser dans le proème une image telle que chaque étape s'assemble aux autres pour former un voyage d'ensemble; il n'est pas douteux qu'il ait échoué, s'il a voulu le faire [...]. Chacune des étapes est compréhensible, ou plus précisément chacune des expressions; mais la peinture tout entière reste remarquablement obscure. On n'arrive pas à tirer une intuition réelle des différentes métaphores. 》

15. Steuben 1981, p. 93-207. Voir par exemple p. 141-142: « Le chemin de Parménide vers la vérité est ainsi un chemin vers la lumière. La vérité ou l'être sont lumière. »

16. Ibidem. p. 149 : « nous admettons que ces réflexions sur les chemins de la Nuit et du Jour ne nous satisfont pas. Mais elles correspondent à une interprétation courante (ou du moins s'en approchent), et nous ne voyons pas bien comment nous pourrions aller plus loin. » La certitude avec laquelle l'auteur porte sa thèse malgré les difficultés qu'elle suscite a cependant ici un fond autobiographique, à savoir l'expérience d'un éveil spirituel à Uppsala en 1954 ; voir là-dessus Steuben 1999, p. 1-29. 
(1988) déplace les chemins de la Nuit et du Jour, qu'elle prétend être distincts l'un de l'autre dans le récit, dans l'Hadès, en s'appuyant explicitement sur les Lamelles d'or ${ }^{17}$, et assimile à titre d'essai la déesse de la révélation à Mnemosyne ${ }^{18}$, sans avoir dans le texte de Parménide de preuve qu'après avoir passé la porte entre ici-bas et l'au-delà, on puisse encore parler de quelque chemin que ce soit.

Peter Kingsley (1999) a mis en relation le voyage, compris dans le sens de l'hypothèse de la nuit, avec des informations sur la vie de Parménide. Par là, il s'appuie surtout sur une inscription hermaïque du $\mathrm{I}^{\text {er }}$ siècle après J.-C. ${ }^{19}$ qui identifie le «Parménéide $e^{20} \gg$ historique comme un membre d'une guilde de guérisseurs d'Élée ${ }^{21}$. Trois membres plus tardifs de cette guilde sont aussi mentionnés sur des inscriptions hermaïques ${ }^{22}$. En s'appuyant sur ces trois dernières inscriptions, Kingsley a pu avancer que l'on pratiquait dans la guilde une thérapie qui consistait en périodes, durant peut-être plusieurs jours, de silence ( $\left.\dot{\eta} \sigma \chi_{i} \mid \alpha\right)$ et de diète réalisées dans une grotte souterraine $e^{23}$. Il va plus loin en montrant que ce «calme » (ívoi $\alpha$ ), auquel le philosophe Parménide doit avoir été initié par son maître pythagoricien

17. Pour les Lamelles d'or, voir l'édition de Bernabé 2005, p. 9-79.

18. Voir particulièrement l'éclairant schéma p. 394-395.

19. On lit sur l'inscription hermaïque découverte à Velia (Pugliese Carratelli 1963,

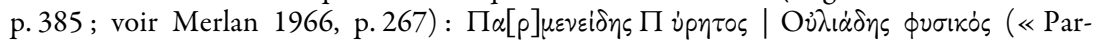
ménéide, fils de Pyrès, ouliade, connaisseur de la nature »); chez Pugliese Carratelli, le $\mu$ après la lacune porte un point, ce qui correspond à la reconstruction de Kingsley 1999, p. 138.

20. En plus du nom, l'emplacement où a été découverte l'inscription (Élée) et le nom du père, Pyrès, que Théophraste indique déjà pour celui du philosophe (chez Alexandre d'Aphrodise, In metaph. 31, 7-14 Hayduck ; voir Diogène Laërce, 9, 21) jouent en faveur de l'identité entre le « Parménéide » de l'inscription et notre philosophe.

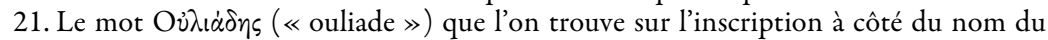
père indique (par analogie avec « asclépiade ») l'appartenance à une guilde vénérant le dieu

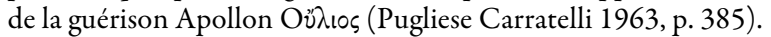

22. Le texte des trois autres inscriptions se trouve chez Pugliese Carratelli 1963, p. 385, et Merlan 1966, p. 267. On peut relier ces inscriptions à celle de Parménide en ce qu'elles

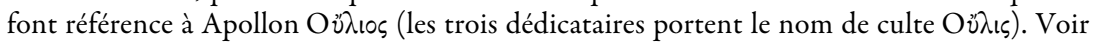
Kingsley 1999, p. 55-60.

23. Kingsley 1999, p. 77-86, résout l'énigme du titre des trois derniers dédicataires,

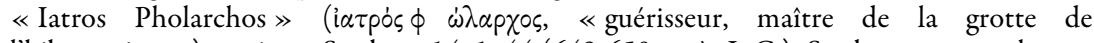
l'hibernation ») en citant Strabon, 14, 1, 44 (649-650 après J.-C.). Strabon compare la pratique de l'incubation à l'hibernation des animaux dans une grotte adéquate ( $\phi \omega \lambda \varepsilon \varepsilon^{\prime}$; le texte

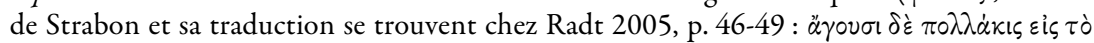

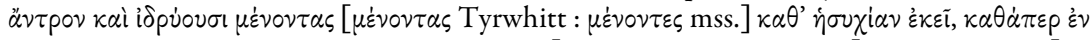

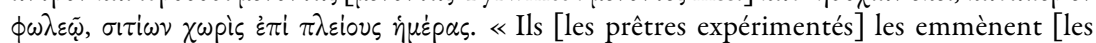
malades] souvent à la grotte et les laissent en cet endroit où ils peuvent alors dans le calme, comme des animaux en hibernation, rester plusieurs jours sans manger ». Pour l'analyse, voir Radt 2009, p. 60-61. 
Ameinias ${ }^{24}$, peut se comprendre à partir de ce contexte comme une absorption méditative ${ }^{25}$. Mais c'est une tout autre histoire d'en tirer quelque chose pour le voyage du poème de Parménide ${ }^{26}$, étant donné la différence de principe entre narrateur intra-textuel et auteur historique. Si le récit, contrairement à la distinction proposée par Burkert (1969), représente une catabase qui laisse de côté la citadelle de la Nuit et mène en fait tout droit vers le royaume souterrain de Perséphone, comme Kingsley l'entend ${ }^{27}$, il fallait d'abord le prouver à partir du texte du proème, avant de l'utiliser pour construire chez Parménide une analogie entre un poème de la catabase et une pratique de la guérison dans les grottes.

Se dessine alors à l'horizon le danger de remplacer l'ancienne aporie (les incohérences de l'hypothèse de la lumière) par une autre : compromettre l'hypothèse de la nuit en introduisant arbitrairement l'image de la catabase et en construisant à partir de là des parallèles avec des sources extérieures.

On observe un certain éloignement par rapport au détail du texte parménidien aussi chez les chercheurs qui se montrent sceptiques à l'égard de l'hypothèse de la nuit. Pour « faire la nulle » entre lumière et nuit (c'est dans l'état des choses le mieux que ce parti puisse atteindre), il semble parfois suffire de faire appel au soi-disant manque de clarté du récit de voyage. Ainsi, Patricia Curd (1998) a affirmé que, malgré la découverte de parallèles apparents avec Hésiode et Homère, il est impossible de se décider entre hypothèse de la lumière et hypothèse de la nuit ${ }^{28}$ :

On a catalogué les influences apparentes d'Homère et d'Hésiode sur le proème, mais on s'accorde peu sur la manière dont le fragment 1 doit être interprété. Il semble clair que le proème a un aspect allégorique, mais on ne voit pas à quoi renvoie l'allégorie. Certains commentateurs ont pensé que le voyage mène à la lumière et à l'illumination, d'autres qu'il se fait sur la route de la demeure de la Nuit, mais malgré leurs protestations, la topographie du

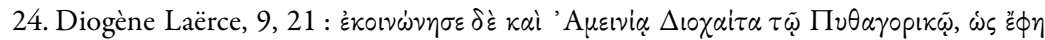

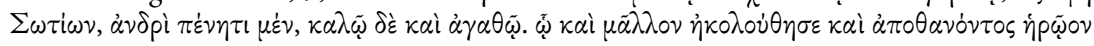

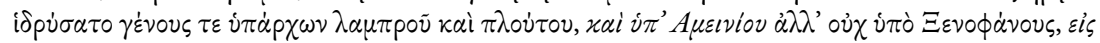

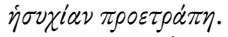

25. Kingsley 1999, p. 173-183.

26. Voir là-dessus la critique de cette tendance générale dans l'interprétation de Kingsley chez Palmer 2009, p. 61, n. 37. Cependant, Palmer partage avec Kingsley le proton pseudos, à savoir de parler à tort de catabase.

27. Kingsley 1999, p. $49-54$ et 93-100 ; voir par exemple p. 94 : « Parménide est descendu aux enfers jusqu’à une déesse qui vit dans le royaume de la mort. Les Grecs l'appellent Perséphone. »

28. Curd 1998, p. 19. 
poème [lire : du proème] est confuse, et je ne pense pas que la destination du kouros soit claire ${ }^{29}$.

Ce non liquet supposerait à proprement parler que les deux hypothèses rendent compte à peu près aussi bien l'une que l'autre du texte parménidien. Mais Curd n'a pas présenté de preuves tirées du texte pour cette ambitieuse hypothèse.

D’un autre côté, John Palmer s'est récemment prononcé pour l'hypothèse de la nuit, et même pour la forme d'origine proposée par Morrison en 1955, c'est-à-dire sans les additions proposées par Sassi et Kingsley ${ }^{30}$. Néanmoins, il ne parvient pas à découvrir comment le récit de voyage aide vraiment à comprendre la révélation ${ }^{31}$. Il en a visiblement été empêché surtout parce qu'il n'a pas vu que le voyage vers la Nuit se divise en deux phases très distinctes : c'est dans cette division que réside en effet, comme nous le montrerons par la suite, le principal indice permettant de déterminer la relation entre doxa et aletheia.

En tout cas, il semble que l'hypothèse de la nuit doive être mieux justifiée et plus précisément formulée avant de se montrer utile pour l'interprétation de la révélation. C'est pourquoi nous devrons par la suite rassembler de la façon la plus complète possible les indices à l'intérieur du texte qui soient pertinents pour se décider entre l'hypothèse de la lumière et celle de la nuit. Sur la base de cette interprétation plus précise du voyage, nous pourrons mettre en évidence non seulement l'identité de la déesse de la révélation, mais aussi la signification de la division en deux du voyage, jusqu'ici passée inaperçue, entre tout d'abord une partie parcourue par le narrateur et son attelage par leurs propres moyens, et ensuite une partie rendue possible par l'escorte divine. Nous ne mentionnerons des parallèles poétiques avec l'élégie et la lyrique ancienne, ainsi qu'avec la théogonie d'Hésiode et l'orphisme ancien, qu'ultérieurement et à fin d'illustration de notre interprétation, et non pour la justifier. Finalement, nous montrerons que l'hypothèse de la nuit, une fois précisée, montre bien de quelle manière le récit de voyage illustre la relation objective entre les deux parties du poème (aletheia et doxa).

29. « The apparent influences of Homer and Hesiod on the proem have been catalogued, but there is little general agreement about how fragment 1 is to be interpreted. It seems clear that the proem has an allegorical aspect, but it is not clear what the allegory is about. Some commentators have seen the journey as one to light and enlightenment, others as a progress along the road to the House of Night, but despite claims to the contrary, the topography of the poem is confusing, and I do not think that it is clear where the kouros has gone. »

30. Palmer 2009, p. 51-61.

31. Ibid.p. 61 : «Cependant, ni cette identification des personnages et des lieux décrits dans le poème, ni aucune autre ne devrait être prise pour la clef qui permet d'interpréter le message qui suit. » 


\section{Le problème et les critères pour le résoudre}

\section{1. Explication de l'hypothèse de la nuit}

Selon l'hypothèse de la nuit, voici ce que le récit de voyage cherche à $\operatorname{dire}^{32}$ : le narrateur serait porté par les cavales qui tirent son char jusqu'au milieu du chemin céleste («chemin du dieu ») que le Soleil pendant la journée et la Nuit après le crépuscule empruntent d'est en ouest. Le point de départ oriental du chemin serait la porte décrite en détail dans la suite du récit; celle-ci serait l'entrée et la sortie de la citadelle de la Nuit, située dans l'au-delà ; le dieu Soleil s'y repose pendant la nuit et ses filles y résident aussi. C'est donc de là que les filles d'Hélios seraient parties pour conduire le narrateur en sécurité le long du chemin de leur père ${ }^{33}$. Dans ce but, elles auraient quitté la citadelle de la Nuit par la porte et arriveraient ainsi « dans la lumière », c'est-à-dire dans notre monde plein de lumière solaire pendant la journée, et plus précisément sur le chemin céleste du Jour et de la Nuit qui part de la porte pour aller vers l'ouest. Elles seraient allées chercher le narrateur sur le chemin divin et seraient revenues avec lui vers l'est jusqu'à la porte, qu'elles auraient passée en même temps que lui, et se retrouveraient à nouveau dans l'obscurité de la citadelle de la Nuit. C'est là que la déesse accueillerait avec bienveillance le narrateur. Morrison a supposé que cette déesse est identique à la maîtresse de la citadelle, c'est-à-dire la déesse de la Nuit; mais les défenseurs ultérieurs de l'hypothèse ne l'ont pas suivi sur ce point, comme nous le verrons par la suite.

En tout cas, les filles d'Hélios, selon l'hypothèse de la nuit, ont effectué non seulement un aller vers la lumière, qui les a conduites, en passant par la porte, de la citadelle de la Nuit dans l'au-delà à l'attelage du narrateur dans notre monde, mais aussi un retour vers l'obscurité, où elles seraient retournées à la porte et l'auraient passée cette fois avec le narrateur et son

32. Voir Morrison 1955, p. 60 : « Le poète est porté par son char jusqu'aux confins de la terre, l'emplacement propre à une catabase (...) En arrivant à l'emplacement traditionnel, il rencontre les filles d'Hélios qui sortent de leur habitation, la demeure de la Nuit, "vers la lumière", c'est-à-dire probablement la lumière du jour. Elles le conduisent au seuil des "portes des trajets de la Nuit et du Jour"; mais pour pouvoir pénétrer dans le monde d'en bas (c'est-à-dire la demeure de la Nuit), elles persuadent d'abord Dikè qui détient les clefs de l'alternance. » Cette présentation de l'hypothèse de la nuit est problématique, d'une part parce que le narrateur n'y est reçu par les filles d'Hélios qu'aux limites du monde, d'autre part à cause du concept de «monde d'en bas » et de « catabase »; voir à ce sujet Burkert 1969 , p. 15 (=13). Ces aspects se laissent cependant corriger sans renverser l'hypothèse en tant que telle.

33. Mansfeld 1964, p. 239 : « Il y a donc deux déplacements : (1) les cavales amènent le poète depuis la terre et le conduisent au chemin de la déesse, (2) les héliades quittent la demeure de la Nuit et prennent la route vers la lumière avant d'accompagner le poète ( $\pi$ po$\left.\lambda_{1} \pi \circ \tilde{\sigma} \sigma \alpha\right)$. Ces déplacements font que le penseur et son attelage d'un côté, les héliades de l'autre, se rencontrent et que le penseur sera conduit plus avant par les filles d'Hélios. » 
attelage, pour revenir dans la citadelle de la Nuit. Le narrateur, en revanche, ne prend part, selon cette hypothèse, qu'au retour dans l'obscurité : les filles d'Hélios sont allées le chercher dans notre monde, plus précisément à un point précis du chemin du Jour et de la Nuit, et l'ont conduit à partir de là jusqu'à la citadelle ${ }^{34}$.

\subsection{Explication de l'hypothèse de la lumiere}

En revanche, l'hypothèse de la lumière ne fait pas de distinction entre un aller et un retour des filles d'Hélios. Elle ne reconnaît au contraire qu'un seul chemin des filles d'Hélios, celui qui conduit de la citadelle obscure de la Nuit directement à la déesse de la vérité, et elle voit dans la destination « vers la lumière » une caractérisation générale de toute la route, même si dans le texte elle ne renvoie directement qu'au départ des filles d'Hélios de la citadelle de la Nuit. C'est pourquoi, selon l'hypothèse de la lumière, le narrateur aurait pris part dans une large mesure à la route des filles d'Hélios « vers la lumière ». Ils auraient trouvé sur leur route commune la porte décrite dans le récit et l'auraient passée ${ }^{35}$. Cependant, en ce qui concerne la fonction et la localisation de la porte, les tenants de l'hypothèse de la lumière se divisent.

Diels (1897) et Fränkel (1930) considéraient le fait que les filles d'Hélios et le narrateur passent ensemble la porte comme le deuxième principal indice en faveur de leur hypothèse (en plus du complément de destination « vers la lumière » que nous venons de mentionner). Pour cela, ils ont dû toutefois ignorer la représentation spontanée selon laquelle il n'y a dans notre monde qu'un seul chemin céleste qui est emprunté d'est en ouest aussi bien par la Nuit pendant la nuit que par le Jour, ou plutôt le Soleil, pendant le jour. Au contraire, Diels et Fränkel ont concrétisé les « trajets $(\kappa \dot{\varepsilon} \lambda \varepsilon v \theta \circ)$ du Jour et de la Nuit » mentionnés dans le texte en deux « chemins du Jour et de la Nuit », situés à différents endroits, et ont alors vu dans la porte un passage entre le «chemin de la Nuit » ici-bas et le «chemin du Jour»dans l'au-delà : les filles d'Hélios auraient quitté la citadelle de la Nuit, parcouru d'abord le chemin de la Nuit, rencontré alors

34. Mansfeld 1964, p. 246 : « Des cavales divines le conduisent sur le chemin après qu'elles sont allées le chercher sur terre. En chemin, il rencontre les héliades, qui ont auparavant quitté la demeure de la Nuit en direction de la lumière et ont relevé le voile de leur tête. C'est-à-dire qu'elles ont quitté la demeure de la Nuit, qui se trouve derrière la porte des trajets du Jour et de la Nuit, et que c'est devenu lumineux. Elles entreprennent de conduire le penseur jusqu'à la porte ; il s'agit pour elles d'un voyage retour. » Cependant, il ne semble pas qu'elles sont allées le chercher sur terre, comme nous le verrons par la suite.

35. Fränkel 1930, p. $157(=161):$ «... tout ce que l'on trouve, c'est : un royaume qui est quitté (celui de la Nuit, de l'attitude sensible et terrestre); une destination (le royaume de la lumière, du Soleil et de la vérité); et entre les deux une porte (la porte de la connaissance). » 
le narrateur, passé avec lui la porte et ainsi atteint le chemin du Jour, sur lequel elles seraient rapidement parvenues à la lumineuse déesse de la vérités. $^{36}$.

Walther Kranz (1916) s'était aussi engagé en faveur de l'hypothèse de la lumière. Mais il était suffisamment impartial pour remarquer les indices clairs dans le texte qui montrent que la porte qui y est décrite doit être la porte de la citadelle de la Nuit et ainsi ne peut pas être la porte d'un palais de la lumière à l'opposé de la Nuit : avec cette observation simple mais fondamentale, Kranz a sans s'en rendre compte posé la première pierre de l'hypothèse de la nuit. Comme cette porte est, sans doute possible, traversée à la fois par les filles d'Hélios et par le narrateur, et comme le lieu que le narrateur atteint en la traversant, selon l'hypothèse de la lumière, ne pouvait en aucun cas être la citadelle de la Nuit, Kranz a dû, pour le salut de cette hypothèse, chercher à leur faire passer la porte dans l'autre direction, c'est-à-dire lire qu'ils quittent la citadelle de la Nuit. C'est pourquoi il a supposé que le narrateur était dès le départ dans la citadelle avec les filles d'Hélios : ils l'auraient quittée ensemble en passant par la porte, et auraient entamé le chemin jusqu'à la déesse de la lumière. Cependant, Kranz a dû accepter que la description du chemin du jour, qui selon lui suit le passage de la porte, ait tout simplement été omise, étant donné que le narrateur explique juste après la description de ce passage comment il a été salué par la déesse. Fränkel (1930) a trouvé le prix payé par Kranz trop élevé, au point de préférer finalement, comme nous l'avons vu, renoncer à la tâche de reconstruire un voyage topographiquement cohérent. En tout cas, à la fin du voyage, selon l'opinion unanime des partisans de l'hypothèse de la lumière, les filles d'Hélios conduisent le narrateur à la déesse de la lumière, qui alors ne peut naturellement pas être la déesse de la Nuit, et pour laquelle on a proposé toutes sortes d'identités différentes ${ }^{37}$.

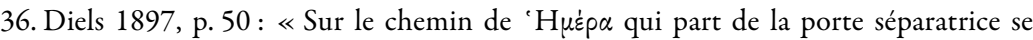
trouve presque immédiatement une habitation, le temple de la déesse. Cette porte doit donc être comprise, selon l'usage de la langue, comme la porte d'une cour (ou d'une ville). »

37. Sextus Empiricus, Adversus Mathematicos, 7, 113 (= Témoignage 136 Coxon) voyait dans la déesse une personnification de la justice (Dike); Deichgräber 1958, p. 663, se demande également : « ou la divinité lumineuse est-elle en fait la déesse Dikè ? », pour affirmer déjà, p. 664 : «Parménide est un homme de Dikè, il est donc accueilli par la déesse Dikè. Elle lui doit de tout lui révéler. » Selon un témoignage de Proclus (In Parm. I, p. 27

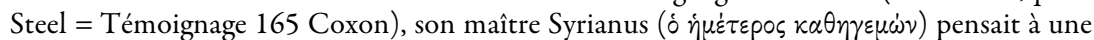
nymphe précise, appelée Hypsipyle («celle qui a une haute porte »); Kranz 1916, p. 1066, a fait entrer en jeu la Muse : « elle est sa déesse ; s'il était poète, nous dirions : sa Muse. » (Voir Fränkel 1930, p. 156.) 


\subsection{Les critères de décision}

L'alternative que nous venons de présenter entre l'hypothèse de la lumière et celle de la Nuit se laisse au fond diviser en quatre sous-alternatives, que l'on peut formuler en questions à double choix. Il est possible de se décider entre d'un côté l'hypothèse de la lumière dans ses deux variantes, de l'autre l'hypothèse de la nuit, si le texte de Parménide apporte une réponse claire à ces quatre questions :

(A) Le narrateur et les filles d'Hélios entament-ils le voyage ensemble, ou se déplacent-ils d'abord de manière séparée ?

(B) Le complément de destination « vers la lumière » vaut-il pour tous les déplacements des filles d'Hélios, ou seulement pour leur départ de la citadelle de la Nuit ?

(C) La porte décrite dans le récit est-elle à comprendre comme à l'opposé de la citadelle de la Nuit, ou faut-il la considérer comme sa porte d'entrée ?

(D) La porte représente-t-elle le passage entre un chemin de la Nuit icibas et un chemin du Jour dans l'au-delà, ou n'y a-t-il qu'un seul chemin céleste qui en part et qui s'étend dans notre monde, parcouru tantôt par la Nuit, tantôt par le Jour ?

Pour admettre la variante de l'hypothèse de la lumière formulée par Diels et Fränkel, il faut considérer comme condition nécessaire et suffisante que pour les questions $\mathrm{B}, \mathrm{C}$ et $\mathrm{D}$, la première possibilité mentionnée soit exacte. Pour admettre la variante défendue par Kranz, il semble que la condition soit que pour les questions A et $\mathrm{B}$ la première possibilité soit exacte, et pour la question C la seconde. Pour admettre l'hypothèse de la nuit au contraire, il semble falloir que pour les quatre questions $\mathrm{A}$ à $\mathrm{D}$, la seconde possibilité soit à chaque fois la bonne.

Il faut à présent examiner le récit de voyage en gardant ces quatre questions à l'esprit. Après s'être décidé entre les deux hypothèses, il faudra alors, dans la mesure du possible, établir l'identité de la déesse de la révélation.

\section{Les étapes de l'action et la topographie dans le texte du récit de voyage}

\subsection{Vers $1-5^{a}$ : les cavales amènent le narrateur au chemin du dieu}

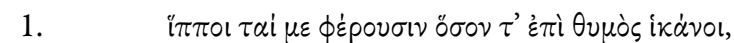

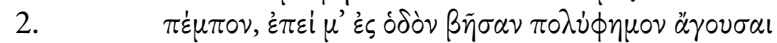




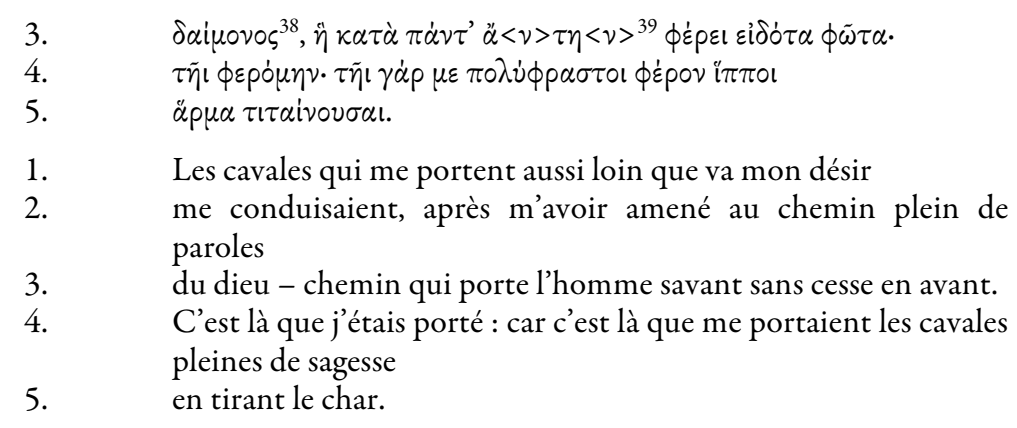

Dans le premier vers, le narrateur présente son attelage : des cavales qui le portent aussi loin ${ }^{40}$ que va son désir ${ }^{41}$. Cette volonté est celle du narrateur lui-même: l'attelage renvoie manifestement à son impetus poético-philosophique ${ }^{42}$. Les vers $2-5^{a}$ expliquent alors que le narrateur a empruntét $e^{43}$ avec son attelage le « chemin du dieu (daimon) ${ }^{44} »$, une fois que ses cavales l'y

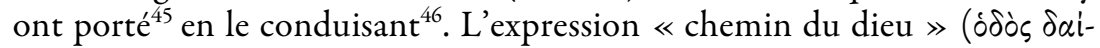

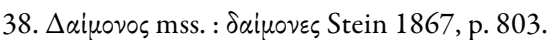

39. $\Pi \dot{\alpha} v \tau^{\prime} \ddot{\alpha}<\nu>\tau \eta<\nu>$ Heyne : $\pi \alpha v \tau \alpha \tau \eta$ mss. (expliqué dans les manuscrits conservés de Sextus tantôt par $\pi \alpha \nu \tau^{\prime} \dot{\alpha} \tau \eta$, tantôt par $\pi \alpha \nu \tau \alpha \tau \eta$, avec des différences dans la position ou même la présence de l'accent). Mutschmann 1914, p. 26, a affirmé qu'il faut lire dans le manuscrit $\mathrm{N}$, le plus ancien de Sextus (Laur. 85,19), $\pi \dot{\alpha} \nu \tau^{\prime} \dot{\alpha} \sigma \tau \eta$, ce qui se trouve déjà dans le texte chez Diels 1912, p. 148 - sans doute parce que Mutschmann avait présenté cette thèse avant de la publier. Cependant, la reproduction photographique du folio 124 verso de ce manuscrit, que l'on trouve chez Coxon 1986, p. 40 sq., montre clairement au début de la huitième ligne $\hat{\eta} \kappa \alpha \tau \dot{\alpha} \pi \dot{\alpha} \nu \tau^{\prime} \ddot{\alpha} \tau \eta$; voir Burkert 1969, p. 5-6 (=5), n. 14, et Coxon 1986,

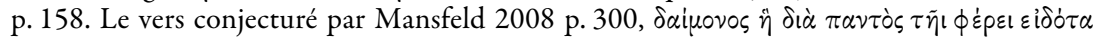
$\phi \tilde{\omega} \tau \alpha$, est insatisfaisant d'un point de vue métrique, étant donné qu'il présente une diérèse absolument proscrite entre le troisième et quatrième pied quand il manque une coupe penthémimère ; voir Martin \& Primavesi 1999, p. 124 (règle I) pour plus de littérature critique.

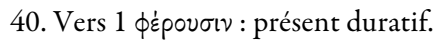

41. Dans les subordonnées qui dépendent d'une principale dont le verbe est un présent désignant un événement régulier, une habitude ou un état permanent, il peut tout à fait y avoir dans la langue épique, au lieu du subjonctif éventuel recommandé par la grammaire académique, un optatif de même nature, comme le montrent les parallèles homériques rassemblés par von Stahl 1907, p. 249. C'est pourquoi il n'est pas besoin de rattacher íkavol (v. 1) à l'imparfait $\pi \dot{\varepsilon} u \pi \circ v$ (v. 2), ce qui est affecté et peu satisfaisant du point de vue du sens.

42. C'est l'avis de Burkert 1969, p. 3 (=3), fin de la note 8 et Coxon 1986, p. 157. Sinon, Mansfeld 1964 p. 231, pense au thymos des cavales: «celui qui dépend complètement de l'escorte divine qui le conduit ne peut aller "aussi loin que va (son propre) désir". » Cependant, le narrateur ne dépend pas «complètement» de l'escorte divine avant d'arriver au chemin divin.

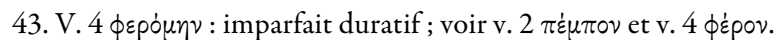

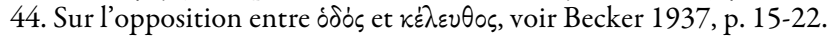

45. V. $2 \beta \tilde{\eta} \sigma \alpha \nu$ : passé aoriste, phrase temporelle renvoyant à une antériorité.

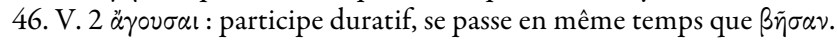


uovos) ne peut pas signifier « chemin vers le dieu ${ }^{47} \gg$, et désigne donc le chemin parcouru habituellement par un dieu ${ }^{48}$. Les dieux qui parcourent habituellement un chemin ne sont pas si nombreux : on peut envisager $N y x$ (Nuit), Selene (Lune), Eos (Aurore), Helios (Soleil). Le vers 9 permettra de répondre. En tout cas, le chemin conduit le savant, d'après Heyne qui complète une lacune de la tradition, sans cesse en avant (v. $3 \alpha \dot{\alpha} \nu \tau \eta \nu)$, c'est-à-dire toujours plus près du but, contrairement aux allées et venues du commun des mortels ${ }^{49}$.

Pour répondre à notre question $\mathrm{A}$ (sur le départ simultané ou séparé du narrateur et des filles d'Hélios), nous allons maintenant montrer que le narrateur doit seulement à son attelage d'avoir atteint le « chemin du dieu », c'est-à-dire à son propre élan poético-philosophique, tandis que l'escorte divine décrite aux vers $5^{\mathrm{b}}-10$ n'entre en jeu que pour parcourir celui-ci.

\subsection{Répondre à la question A: le narrateur et les filles d'Hélios entament-ils le voyage ensemble, ou se déplacent-ils d'abord de manière séparée?}

L'idée que le départ décrit dans les vers $1-5^{a}$ pourrait être commun au narrateur et aux filles d'Hélios est née de la correction introduite dans le texte par Heinrich Stein (1867) : au lieu du génitif singulier daimonos ( $\delta \alpha i-$

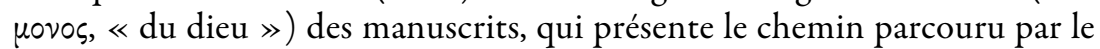
narrateur comme celui habituellement emprunté par un dieu, il met au

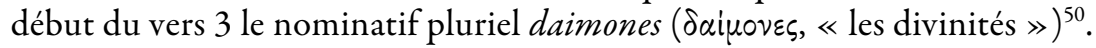
Cette correction a pour conséquence que ce ne sont plus les cavales qui por-

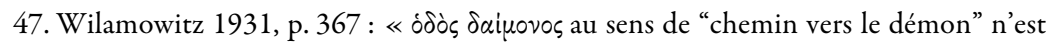
pas du grec. »

48. Pfeiffer 1975 a raison sur ce point : « les exemples homériques nous apprennent que là où un chemin et une personne (au génitif) sont associés, cette personne doit se trouver sur le chemin. »

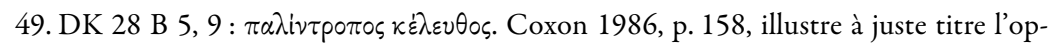

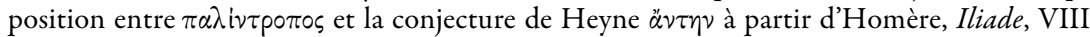

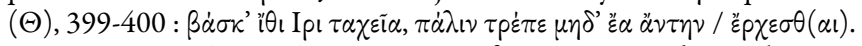

50. Stein 1867 , p. $769-770$ et 803 , a forgé ce qui est devenu la version canonique quand Wilamowitz l'a admise, même si celui-ci analyse la relation entre les chevaux et le conducteur d'abord d'un point du vue hippique plutôt que de prendre en compte les implications poétiques (Wilamowitz 1899, p. 204) : « [...] celui qui dit de lui-même que ses cavales l'ont porté au chemin se présente comme un mauvais exemple. Mais dans la répétition parallèle v. 5 et dans le discours de la déesse v. 24, ce sont les héliades qui conduisent :

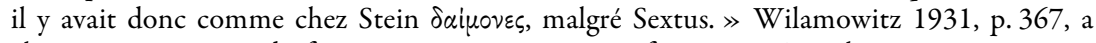
alors jugé opportun de fournir une tout autre justification, guère plus convaincante, au maintien de la conjecture de Stein : il rejette désormais l'expression des manuscrits óò̀v [...]

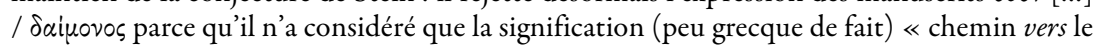
dieu », et non le fait que l'expression telle qu'elle est transmise, avec la construction usuelle (« chemin $d u$ dieu »), montre indubitablement que le chemin est parcouru habituellement par un dieu. 
tent le narrateur au chemin, mais les daimones introduits par Stein ${ }^{51}$, qui devraient alors être des « déesses », puisqu'ils deviendraient assurément féminins à cause du participe à la fin du vers $2(\ddot{\alpha} \gamma \sigma v \sigma \alpha l)$. Et ces « déesses » ne pourraient alors qu'être les jeunes filles, introduites, dans le texte tel qu'il est transmis, seulement au vers $5^{\text {b }}$, et identifiées au vers 9 comme filles du dieu du Soleil Hélios. Ainsi, alors que selon le texte transmis, en toute probabilité, les filles d'Hélios n'arrivent à l'attelage du narrateur et n'entreprennent de l'escorter qu'après que les cavales l'ont porté au chemin du dieu (du Soleil), il semblerait, selon la correction de Stein, que le narrateur ait été dès le début, à son départ, avec les filles d'Hélios. La distinction fondamentale entre le moment où le char atteint le chemin, d'un côté, et celui, de l'autre, où l'aide divine lui est accordée pour le parcourir est alors effacée.

Kranz (1916) cependant a vu dans la correction de Stein une opportunité pour consolider l'hypothèse de la lumière, malgré un emplacement de la porte qui ne va pas dans son sens. Il est en effet arrivé à la conclusion que la porte qui est traversée selon les vers 15-21 par les filles d'Hélios en même temps que le narrateur doit, sur la base de la description des vers 1114, être identifiée à la porte de la citadelle de la Nuit. Mais l'hypothèse de la lumière, qui suppose qu'ils passent la porte en direction de la lumière, n'est compatible avec cette localisation que si l'on suppose que le narrateur et les filles d'Hélios ont quitté ensemble cette citadelle par la porte. La conjecture de Stein lui a semblé ouvrir cette possibilité : d'après Kranz, le récit commence au milieu du voyage commun du narrateur et des filles d'Hélios (v. 1-10), puis fait un retour en arrière pour évoquer leur passage commun de la porte au début du voyage (description de la porte : v. 11-14; passage de la porte : v. 15-21), et enfin, après ce flash-back ${ }^{52}$, il reviendrait aux salutations de la déesse qui ont lieu en fait à la fin du voyage (v. 22 sq. $)^{53}$. Kranz a repris cette interprétation en 1934 dans sa réédition des Fragmente der Vorsokratiker, où elle a été réimprimée sans changement jusqu’à aujourd'hui ${ }^{54}$. Néanmoins, la conjecture de Stein paraît complètement arbitraire

51. H.-J. Mette est allé encore plus loin, en voulant changer, dans une note de son propre exemplaire des Fragmente der Vorsokratiker, le i $\pi \pi 0$ que nous avons au vers 1 en

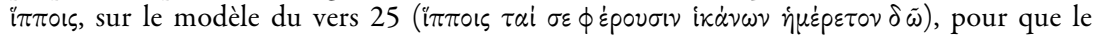
$\delta \alpha i \mu \circ v \varepsilon \varsigma$ conjecturé par Stein puisse servir de sujet au $\pi \dot{\varepsilon} \mu \pi \circ v$ du vers 2 .

52. D'un point de vue narratologique, il s'agirait d'une analepse interne.

53. Kranz 1916, p. 1161 : « Mais à la fin de la description de ce voyage sont introduites

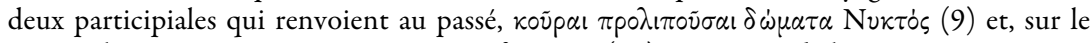
même plan, $\dot{\omega} \sigma \dot{\alpha} \mu \varepsilon v \alpha \iota ~ \kappa \rho \dot{\alpha} \tau \omega \nu \ddot{\alpha} \pi \circ \chi_{\varepsilon} \rho \sigma \grave{\text { i }} \kappa \alpha \lambda \dot{\nu} \pi \tau \rho \alpha \varsigma(10)$, et à partir de là un nouveau passage raconte comment les filles d'Hélios ont quitté cette demeure de la Nuit (11-21). »

54. Diels-Kranz 1934, p. 228 ; apparat à la ligne 17 : « Nous divisons le récit en trois parties : 1. le voyage v. 1-10 / 2. (en retour en arrière) le départ v. 11-21 / 3. l'arrivée v. 22-32 (contrairement à Fränkel). » Kranz n'y a rien changé dans les compléments de la sixième édition (1951). 
étant donné que l'image d'un « chemin du dieu » est non seulement loin d'être incompréhensible en elle-même, mais de plus étroitement liée au fait que les filles d'un dieu jouent le rôle de passeur sur ce chemin, comme nous le verrons par la suite. L'hypothèse de Stein entraîne dans sa chute la variante de l'hypothèse de la lumière proposée par Kranz depuis 1916. Le narrateur commence son voyage sans les filles d'Hélios, et atteint le chemin divin sans leur aide.

\subsection{Vers 5b-10 : les filles d'Hélios conduisent l'attelage le long du chemin du} Soleil

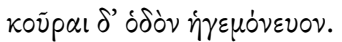

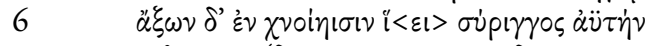

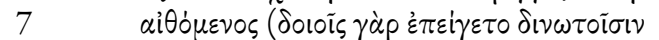

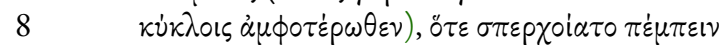

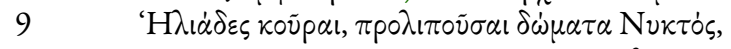

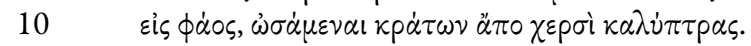
Mais de jeunes filles me montraient la route : l'essieu dans les moyeux émettait un son comme une syrinx, quand il s'embrasait (car il était emporté par deux roues

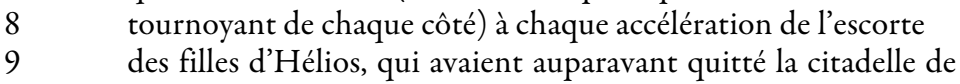 9 des fill
venant ainsi vers la lumière, et relevant alors de leurs mains les voiles de leurs têtes.

Au vers $5^{\text {b }}$, les jeunes filles qui précèdent l'attelage du narrateur sur le chemin divin sont mentionnées pour la première fois ${ }^{55}$. Le prédicat en

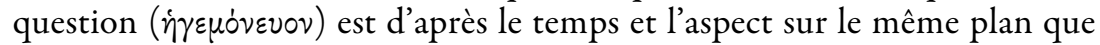
le prédicat du vers 4 ( $\phi \dot{\varepsilon} p o v)$, qui renvoie au voyage de l'attelage sur le chemin divin. D'autre part, depuis que tous ont rejeté la conjecture mal-

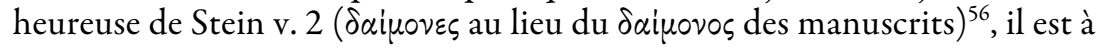
nouveau clair que les jeunes filles n'ont pas contribué à amener le narrateur au chemin du dieu. À partir de là, il s'ensuit directement qu'elles conduisent l'attelage depuis qu'il s'avance sur le chemin divin.

Alors, le vers 9, où les jeunes filles sont identifiées comme filles $d u$ dieu du Soleil Hélios, nous montre que c'est ce dieu qui a la charge du chemin parcouru par le narrateur. En effet, pourquoi est-ce que celles-ci se sentiraient responsables de la sécurité sur le « chemin du dieu » (v. 2-3 ódòv...

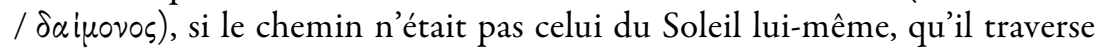

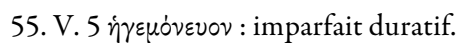

56. Pour un retour à la leçon des manuscrits, voir par exemple Tarán 1965, p.7; Hölscher 1969, p. 10 ; Heitsch 1974, p. 8 ; Gallop 1984, p. 48 ; Coxon 1986, p. 45 ; O’Brien 1987, p. 3. 
pendant la journée d'est en ouest ? Mais nous devons ajouter que pendant la nuit, le chemin appartient à la Nuit : c'est la même route qui sert aux

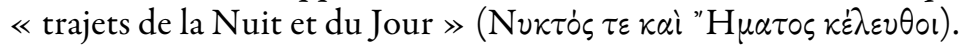

Ainsi, on peut voir la relation suivante entre les cavales d'un côté et les jeunes filles de l'autre : les cavales seules ont amené le narrateur et son char jusqu'au chemin céleste qui est en fait réservé pendant la journée au Soleil. Mais après que l'attelage du narrateur a atteint ce chemin, les filles d'Hélios ont pris la responsabilité d'escorter en sécurité l'attelage, et ce manifestement par crainte des dangers qui menaceraient le narrateur dans sa route sur le chemin du Soleil - on ne peut que penser au voyage comparable mais fatal de leur frère Phaéton ${ }^{57}$. Néanmoins, il ne faut pas abuser du parallèle entre le narrateur et Phaéton: le narrateur va certes sur le chemin du Soleil, comme Phaéton, mais contrairement à lui il ne conduit pas le char du Soleil, au contraire de ce que John Palmer a récemment affirmé $^{58}$, mais il conduit le char habituel du poète, comme le montre le vers 1.

En tout cas, les filles d'Hélios se sont rendues devant l'attelage du narrateur et à plusieurs reprises poussent ses cavales à accélérer ${ }^{59}$, ce qui fait que les essieux, poussés par des roues tournoyant des deux côtés ${ }^{60}$, émettent à chaque fois un son flûté ${ }^{11}$. Les vers 9-10 expliquent d'où les filles d'Hélios sont venues pour accomplir leur tâche : elles ont quitté la citadelle de la

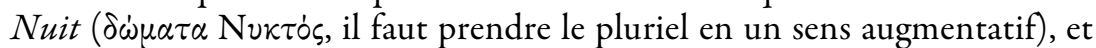
ont alors relevé leur voile.

3.4 Retour à la question A : est-ce que le narrateur et les filles d'Hélios entament le voyage ensemble, ou est-ce qu'ils se déplacent d'abord de manière séparée?

Le fait que les filles d'Hélios quittent la citadelle de la Nuit et qu'elles relèvent leur voile est à chaque fois décrit avec un participe aoriste (v. 9

57. Sur le mythe de Phaéton, voir Burkert 1969, p. 6-7 (=6).

58. Palmer 2009, p. 56 : «En identifiant ses guides comme les filles d'Hélios, Parménide montre bien que son char et les cavales qui le tirent appartiennent à Hélios. » Devraiton penser que le narrateur sort régulièrement de chez lui avec l'attelage du dieu Soleil ? Que fait Hélios pendant ce temps?

59. V. $8 \sigma \pi \varepsilon p x o i \alpha \tau o:$ optatif itératif. Il ne faut pas comprendre qu'il y a eu plusieurs voyages du narrateur et que les filles d'Hélios se sont à chaque fois empressées de le conduire (Wilamowitz 1899, p. 203 : «Ce n'était pas le premier voyage où il parvenait ici et où ses essieux chauffaient, car à chaque fois que les héliades l'ont escorté, la vitesse était la même. »; Fränkel 1930, p. 155 n. 3 ; Deichgräber 1958, p. 656 : «à chaque fois que les jeunes héliades se hâtaient pour m'escorter. »), mais plutôt que les filles d'Hélios, dans le voyage du narrateur dont il est question ici, accélèrent plusieurs fois le rythme de l'escorte, et donc le voyage. Voir Burkert 1969, p. 3 (=3) n. 8.

60. V. 7 ध่ $\pi \varepsilon i \gamma \varepsilon \tau 0:$ imparfait duratif.

61. V. $6 i<\varepsilon l>$ : imparfait duratif. 
$\pi p \circ \lambda ı \pi \circ \tilde{\sigma} \sigma \alpha$ et v. $10 \omega \dot{\omega} \sigma \dot{\alpha} \mu \varepsilon \nu \alpha \iota)$. Étant donné l'emploi général en grec du participe aoriste, on ne peut douter, vu que le récit jusqu'ici s'inscrivait dans le duratif ${ }^{62}$, que ces deux participes renvoient à une antériorité ${ }^{63}:$ les filles d'Hélios ont quitté la citadelle de la Nuit et relevé leur voile avant de servir de passeur au narrateur dans son voyage sur le chemin du Soleil. Les participes aoristes supposent une inscription dans le passé de l'arrivée de l'escorte des filles d'Hélios, comme au vers 2 la subordonnée à l'aoriste avec

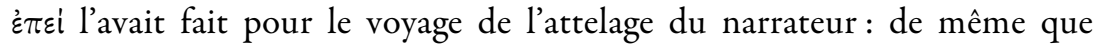
celui-ci a commencé son voyage sans les filles d'Hélios, de même celles-ci ont quitté la citadelle de la Nuit sans sa compagnie, mais dans l'intention d'aller le chercher pour l'aider et l'emmener.

\subsection{Répondre à la question B : le complément de destination « vers la lumière » vaut-il pour tous les déplacements des filles d'Hélios ou seulement pour leur départ de la citadelle de la Nuit?}

Le fait que les filles d'Hélios aient quitté la citadelle de la Nuit seules et avant de rejoindre le narrateur a des conséquences sur l'interprétation de l'important complément de destination: «vers la lumière » (v. $10 \varepsilon$ is $\phi \dot{\alpha} \circ \varsigma)$. La signification de cette expression est cruciale dans l'alternative entre l'hypothèse de la lumière et celle de la Nuit en ce qu'il s'agit du seul passage dans tout le récit où il est question d'un déplacement « vers la lumière ». Cependant, ce complément de destination se trouve dans un enjambement juste après la participiale qui décrit les filles d'Hélios qui

62. L'examen des huit premiers vers a montré que le voyage est raconté avec des imparfaits duratifs, avec trois exceptions : pour l'une, la relation entre le narrateur et les cavales est marquée comme habituelle, antérieure à l'événement, par le choix du présent duratif (v. $1 \phi \dot{\varepsilon}$ pov $\sigma$ ). Pour la seconde, la phrase dans laquelle Parménide raconte que le narrateur est mené à la route (v. $\left.2 \dot{\varepsilon} \pi \varepsilon \dot{l} \mu^{\prime} \dot{\varepsilon} \varsigma \delta \delta \delta o ̀ \nu \beta \tilde{\eta} \sigma \alpha \nu\right)$ est à l'aoriste; la conjonction temporelle (v. $2 \dot{\varepsilon} \pi \varepsilon i$ ) ainsi que le contexte établissent sans doute possible que cet aoriste sert à exprimer une antériorité effective : le narrateur a en effet dû d'abord être mené à la route avant de pouvoir la parcourir. Pour la troisième, le chemin du dieu est caractérisé dans un

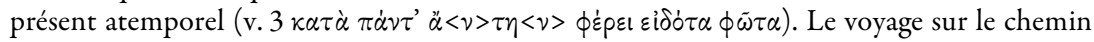
du dieu est pour sa part inscrit sans exception dans le cours d'un événement passé durable. Voir là-dessus Burkert 1969, p. 7-8 (= 6-7).

63. Il est certes vrai qu'en grec, c'est seulement à l'indicatif que les verbes renvoient explicitement au passé; c'est pourquoi il n'y a d'augment qu'à l'indicatif. Mais s'il est en théorie possible que le participe du radical aoriste ponctuel désigne une action contemporaine de celle du prédicat de la principale, dans les faits, comme le montrent les exemples de la grammaire, cela se limite aux cas où le prédicat de la principale est aussi à l'aoriste, de sorte qu'il s'agit de la simultanéité d'un point avec un autre, par exemple « $\varepsilon \tilde{y} \gamma^{\prime} \dot{\varepsilon} \pi$ in $\dot{\alpha} \nu \alpha \mu \nu \eta \dot{\sigma} \sigma \varsigma \mu \varepsilon \gg$ (Platon, Phédon, 60c9). Par contre, si le prédicat de la principale est duratif, le participe aoriste renvoie alors à une antériorité : il contredirait directement l'aspect ponctuel et perfectif de l'aoriste si l'action en question était tout aussi inachevée et encore en cours que l'action de la principale qui s'exprime avec un verbe à l'imparfait. Voir Schwyzer/Debrunner, p. 300 sq. 


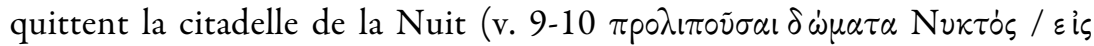
фáos) et est donc au milieu des deux participes qui renvoient à ce départ (v. $9 \pi p \circ \lambda ı$ เ moment où les filles d'Hélios quittent la citadelle de la Nuit, avant le voyage commun avec le narrateur ${ }^{64}$ : celles-ci arrivent «à la lumière », c'est-à-dire au monde illuminé de jour par la lumière du Soleil, quand elles quittent la forteresse de la Nuit pour faire escorte au narrateur sur le chemin du dieu Hélios. Cela est confirmé par le fait qu'en même temps qu'elles sortent de la citadelle, elles relèvent leur voile.

De plus, la préposition « $\varepsilon i \zeta$ » montre qu'il ne s'agit pas ici d'une destination encore à atteindre, comme Hölscher (1969) et Heitsch (1974) l'ont suggéré dans le sens de l'hypothèse de la lumière avec leur traduction « dem Lichte $z u »$ («en direction de la lumière »), mais qu'elle vient d'être atteinte : en quittant la citadelle de la Nuit, les filles d'Hélios sont allées tout de suite dans la lumière, avant de faire escorte au narrateur ${ }^{65}$. À partir de là, on ne peut plus estimer que la suite du voyage qui se fait en commun avec le narrateur sur le chemin du Soleil soit une portion de la route vers la lumière : au contraire, les filles d'Hélios se trouvaient déjà complètement dans ce qu'on appelle ici lumière lorsqu'elles ont entrepris d'escorter le narrateur, et on peut juste se demander si le voyage commun qui suit reste dans le domaine de la lumière, ou s'ils font demi-tour à partir de là vers la nonlumière, l'obscurité.

Il résulte de cette analyse une conséquence qui mine l'hypothèse de la lumière : le seul passage du proème qui présente un déplacement à destination « de la lumière » ne renvoie absolument pas au voyage commun des filles d'Hélios et du narrateur. Les tenants de cette hypothèse ont naturellement cherché à s'y dérober. En plaçant une ponctuation juste avant

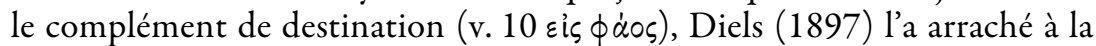
participiale, qui renvoie à un temps antérieur au voyage, pour le raccorder au dernier verbe principal rencontré, qui se trouve deux vers auparavant, v. 8

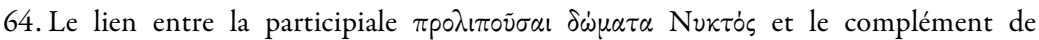

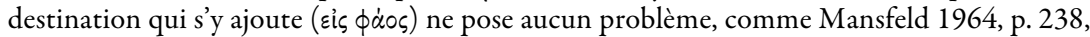
l'a expliqué de façon précise, en s'appuyant sur Kühner/Gerth, Syntax, I, $\$ 447$ : « un verbe implique le concept du mouvement qui le suit quand la préposition eis est avec l'accusatif,

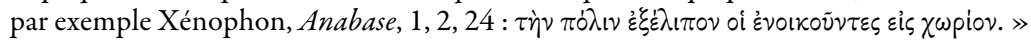

65. Contrairement à « $\pi$ pós », la préposition « $\varepsilon i \varsigma$ » désigne, dans un déplacement achevé, non pas simplement un rapprochement, mais une arrivée quelque part (angl. into); voir LSJ s. v. zic I 1 a. 
( $\sigma \pi \varepsilon \rho \chi o i \alpha \tau \circ \pi \dot{\varepsilon} \mu \pi \varepsilon \iota \nu)^{66}$; Mansfeld (1964) l'a critiqué avec raison ${ }^{67}$. Hölscher et Heitsch ont alors dans leur traduction non seulement extrait le complément de destination de son contexte à l'aide de virgules, comme Diels dans son édition, mais aussi laissé tomber la différence de temps entre le voyage commun raconté à l'imparfait et le départ antérieur des filles d'Hélios $^{68}$. Ils avaient donc besoin d'un moyen vraiment contestable pour élargir le complément de destination « vers la lumière » afin qu'il ne concerne pas seulement le départ des filles d'Hélios mais aussi leur voyage commun avec le narrateur.

En conclusion, nous ne pouvons qu'affirmer que le déplacement des filles d'Hélios « vers la lumière » s'achève quand elles quittent seules la citadelle de la Nuit, avant qu'elles entreprennent d'escorter le narrateur.

\subsection{Vers 11-14: la porte des trajets de la Nuit et du Jour}
11

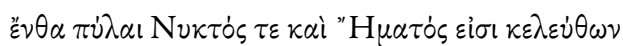

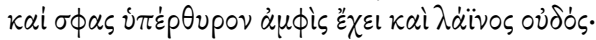

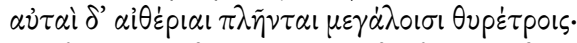

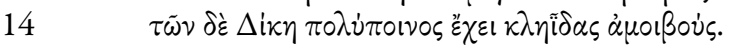
11 C'est là que se trouve la porte des trajets de la Nuit et du Jour

66. Diels 1897 , p. 28-29, ponctue les vers $8^{\mathrm{b}}-10$ de cette manière: ö $\tau \varepsilon$ $\sigma \pi \varepsilon p \chi 0 i \alpha \tau o$

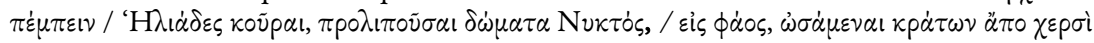
$\kappa \alpha \lambda \dot{\pi} \pi \tau$ pas, et traduit en conséquence : « quand les héliades qui avaient quitté la demeure de la Nuit et rejeté alors le voile de leurs têtes hâtèrent le voyage vers la lumière. »

67. Mansfeld 1964, p. 238 n. 1: «Eis ф́áos se trouve entre deux part. aor. et en est extrait. Mais l'ordre des mots est alors très contourné. » ; voir par la suite Burkert 1969, p. 7 (=6-7); Palmer 2009, p. 53.

68. Hölscher 1969, p. $11:$ «...wenn schleuniger sich sputeten die Sonnenmädchen, mich voranzufahren, hinter sich das Haus der Nacht, dem Lichte zu, und von den Häuptern mit Händen die Schleier aufschlugen » (« [...] quand les filles d'Hélios se hâtaient encore plus de me conduire, avec la demeure de la Nuit derrière elles, en direction de la lumière, et rabattaient de leurs mains le voile de leur tête » On remarque la simultanéité de «se hâtaient » et 《rabattaient » et l'isolement de «en direction de la lumière »); Heitsch 1974, p. 9: «...so oft sich die Heliadenjungfrauen beeilten zum Geleit, verlassend die Behausung der Nacht, zum Licht, vom Haupte den Schleier mit den Händen zurückschlangend. » («[...] quand les filles d'Hélios me conduisaient plus vite, laissant derrière elles la demeure de la Nuit, en direction de la lumière, en poussant de la main le voile de leur tête ». On remarque les participes simultanés « laissant » et « poussant » et l'isolement de « en direction de la lumière »). On retrouve la même imprécision grammaticale dans le traitement des participes, bien qu'avec un autre sens, chez Deichgräber 1958, p. 656, qui semble déjà prendre le «se hâtaient » itératif (en s'appuyant sur Wilamowitz 1899, p. 203) comme antérieur à «l'escorte» effective : « ...so oft sich die Heliadenjungfrauen beeilten zum Geleit, verlassend die Behausung der Nacht, zum Licht, vom Haupte den Schleier mit den Händen zurückschlagend» («[...] à chaque fois que les jeunes héliades se hâtaient pour m'escorter, laissant l'habitation de la Nuit, vers la lumière, en rejetant de leurs mains le voile de leur tête ».) 
12 et un linteau l'encadre ainsi qu'un seuil de pierre.

13 La porte elle-même, faite d'éther, est remplie par de grands battants.

14 La clef pour les ouvrir et les fermer est détenue par Dikè pleine d'amendes.

Aux vers 11-14, le récit a recours à une description (ekphrasis) formulée au présent : il y décrit la grande porte par laquelle la Nuit comme le Jour passent pour effectuer leur trajet ${ }^{69}$. Au début de la description, nous trouvons un indice important pour localiser la porte, que nous allons examiner plus précisément dans le cadre de notre question C. Par contre, nous pourrons plus aisément élucider des questions comme celle de la localisation de la route de la Nuit et du Jour ou celle du rôle de la justice (Dike) en les reliant au franchissement de la porte par le narrateur, qui sera raconté dans le passage suivant (v. 15-23).

3.7 Répondre à la question $C$ : la porte décrite dans le récit est-elle à comprendre comme à l'opposé de la citadelle de la Nuit, ou faut-il la considérer comme sa porte d'entrée?

La description de la porte est introduite par l'adverbe démonstratif local « là » $(v .11$ हैv $\theta \alpha)$. Un tel « là » renvoie à ce qui vient d'être dit. Il en ressort qu'il ne faut pas chercher à appuyer cet adverbe sur le récit par le narrateur de son départ vers le chemin du dieu Soleil (v. $1-5^{\mathrm{a}}$ ), ni sur la description de son voyage sur ce chemin à la suite des filles d'Hélios (v. $\left.5^{\mathrm{b}}-8^{\mathrm{a}}\right)$, mais en regardant juste avant, quand les filles d'Hélios ont quitté la

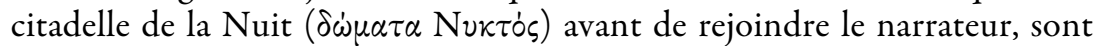
alors arrivées «dans la lumière » (zis $\phi \dot{\alpha} \circ \varsigma)$ et ont relevé le voile de leur tête (v. $\left.8^{\mathrm{b}}-10\right)$. Les lieux décrits juste avant le démonstratif anaphorique « là » sont tout d'abord la solide « citadelle de la Nuit », ensuite l'indication très générale «vers la lumière » qui renvoie à la lumière du jour terrestre. Si l'on compare les deux, on doit admettre avec Kranz (1916) qu'en toute objectivité, seule la citadelle de la Nuit peut être considérée comme le lieu de la $\operatorname{porte}^{70}$. De plus, les vers $8^{\mathrm{b}}-10$ ne parlent pas de la citadelle de la Nuit en

69. Pour le sens originel verbal et complètement transparent de $\kappa \dot{\varepsilon} \lambda \varepsilon v-\theta_{\circ}$ (pousser $\kappa \varepsilon \lambda \varepsilon \dot{\varepsilon เ \nu ~-~ l e ~ c h a r ~ p o u r ~ l e s ~ c h e v a u x), ~ v o i r ~ B e c k e r ~ 1937, ~ p . ~ 7-14 . ~}$

70. Kranz 1916, p. 1160 : « Pour qu' 'ै $\nu \theta \alpha$ ne reste pas en suspens, il faut chercher une description locale dans ce qui précède; on n'en trouve qu'une seule: la demeure de la Nuit. » Ce que Deichgräber 1958, p. 658-659, avance contre l'observation de Kranz est inapproprié : « nous ne devons pas interpréter है $\nu \theta \alpha$ comme renvoyant à un lieu concret mais comme l'indication d'un endroit quelconque sur le chemin de Parménide. Le हैं $\theta \alpha$ est employé de la même manière que ce que nous rencontrons si souvent dans la poésie de catalogue hésiodique et post-homérique, sans que nous trouvions un emplacement précis auquel renvoie ce mot. » Le parallèle présenté par Deichgräber, à savoir la série, sous forme de catalogue, d'indications avec है $2 \theta \alpha$, n'en est pas un; car il n'y a dans notre passage qu'un 
général, mais plus particulièrement du fait de quitter cette citadelle, de sorte qu'on ne peut alors penser qu'à une porte qui permette de sortir. Pour l'instant donc, tout confirme que la porte décrite aux vers 11-14 est celle de la citadelle de la Nuit.

Ce résultat, insignifiant en soi, est cependant d'une importance considérable pour l'alternative entre hypothèse de la lumière et hypothèse de la nuit. À l'intérieur du récit dans son ensemble, la description de la porte occupe une position charnière : elle s'appuie d'abord sur le moment où les filles d'Hélios quittent la citadelle de la Nuit, mais mène ensuite à la description de la traversée commune de la porte par le narrateur et celles-ci. Si la porte est bien celle de la citadelle de la Nuit, et si, comme nous venons de le montrer, les filles d'Hélios ont quitté celle-ci seules (voir notre traitement de la question A), alors, lorsqu'ils traversent ensemble la porte, il ne peut s'agir pour les filles d'Hélios que d'un retour à la citadelle de la Nuit - retour où elles amènent le narrateur avec elles. Nous démontrerions ainsi que le texte mentionne un aller vers la lumière, puis un retour vers l'obscurité, mais aussi que la participation du narrateur se limite au chemin vers l'obscurité. Ce résultat, qui est le prélude à une réfutation de l'hypothèse de la lumière, reste pourtant incomplet tant que nous n'avons pas examiné la deuxième prémisse de celle-ci, à savoir la concrétisation des « routes de la Nuit et du Jour » en deux chemins différents et situés de part et d'autre de la porte (question $\mathrm{D}$ ).

3.8 Vers 15-23: le narrateur est conduit à travers la porte et accueilli par la déesse

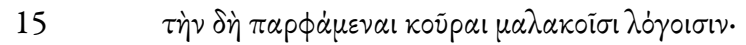
$16 \pi \varepsilon \tilde{\sigma} \sigma \alpha \nu \dot{\varepsilon} \pi \iota \phi \rho \alpha \delta \dot{\varepsilon} \omega \varsigma, \ddot{\omega} \varsigma \sigma \phi ı \nu \beta \alpha \lambda \alpha \nu \omega \tau \dot{\partial} \nu$ ó $\chi \tilde{\eta} \alpha$

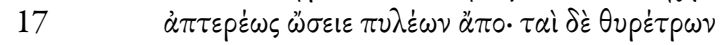

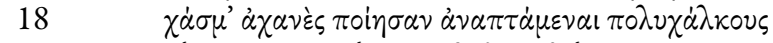

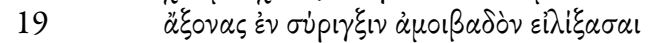

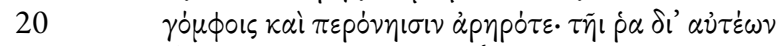

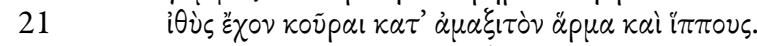

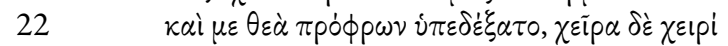

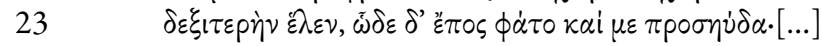
15 Les jeunes filles s'exprimèrent alors avec de douces paroles
16 et la persuadèrent habilement de retirer rapidement de la porte, pour elles,
17 la barre munie de chevilles. Ses battants volèrent et créèrent
18 une ouverture immense en faisant tourner les montants

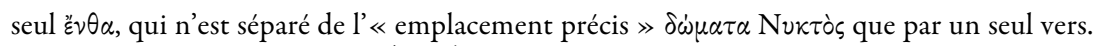
En outre, Burkert 1969, p. $12(=11)$ n. 27 a aussi relativisé l'affirmation de Deichgräber qu'un है $v \theta \alpha$ répété chez Hésiode serait peu précis dans sa localisation. 
19 de bronze épais, garnis de clous et d'agrafes,

20 l'un vers l'autre dans les gonds. Alors, les jeunes filles

21 firent traverser le char et les chevaux en suivant l'allée.

22 Et la déesse m'accueillit avec bienveillance; de sa main

23 elle prit ma main droite, s'adressa à moi et dit ceci : [...]

À partir du vers 15 , le récit reprend ${ }^{71}$ pour expliquer comment les filles d'Hélios font passer l'attelage du narrateur par la porte des trajets de la Nuit et du Jour décrite auparavant ${ }^{72}$. Elles convainquirent assez la gardienne (Dike) pour qu'elle leur ouvre la porte, et elles firent passer la porte $\left(\delta \iota^{\prime} \alpha \dot{\tau} \tau \dot{\varepsilon} \omega \nu\right)$ au char et aux chevaux par l'allée. Le voyage s'achève de toute évidence avec le passage de la porte ; car juste après la déesse accueille avec bienveillance le narrateur ${ }^{73}$. Cette analyse permet d'examiner la question qui nous reste, à savoir comment il faut comprendre l'expression « porte des trajets de la Nuit et du Jour ».

3.9 Répondre à la question D : la porte représente-t-elle le passage entre un chemin de la Nuit ici-bas et un chemin du Jour dans l'au-delà, ou n'y a-t-il qu'un seul chemin céleste qui en parte et qui s'étende dans notre monde, parcouru tantôt par la Nuit, tantôt par le Jour?

L'identification de la porte comme celle des trajets du Jour et de la Nuit est le point du récit où les difficultés de l'hypothèse de la lumière sont les plus apparentes. D'un côté, celle-ci trouve un de ses deux principaux appuis dans l'idée vite construite et par la suite jamais vraiment remise en question que la Nuit voyage sur un autre chemin que le Jour : le chemin de la Nuit est de ce côté de la porte et celui du Jour de l'autre. La porte servirait alors à séparer le chemin de la Nuit et celui du Jour, et le narrateur et les filles d'Hélios se trouveraient d'abord sur le premier pour ensuite, en passant la porte, parcourir le second par lequel ils arriveraient enfin à la lumineuse déesse de la vérité ${ }^{74}$.

71. Le temps du récit est à présent essentiellement, avec une exception v. 21, l'aoriste, par opposition aux formes de passé duratif de la première partie.

72. Que la porte traversée ici soit la même que la « porte des trajets de la Nuit et du Jour » décrite auparavant se déduit directement du fait que le récit commence par un pronom démonstratif (v. $15 \tau \dot{\eta} v)$, qui renvoie clairement à Dike, c'est-à-dire à la gardienne présentée au v. 14.

73. La thèse de Kranz 1916 selon laquelle le voyage du narrateur et des filles d'Hélios vers la déesse ne fait que commencer avec le passage de la porte, même si cela est omis dans le récit, n'était dès le début qu'une solution ad hoc qui, comme nous l'avons vu, est de toute façon devenue caduque avec la suppression de la correction ratée de Stein au vers 3 : les filles d'Hélios ne quittent pas la citadelle de la Nuit avec le narrateur, mais sans lui.

74. Voir Diels 1897, p. 50 : « Sur le chemin d' 'H $\mu \dot{\varepsilon}$ pa qui part de la porte séparatrice se trouve presque immédiatement une habitation, le temple de la déesse. » 
Cette interprétation échoue cependant ne serait-ce que sur l'idée que la porte, comme nous l'avons vu, se trouve à la fin du chemin parcouru par le narrateur avec les filles d'Hélios. Le texte ne signale pas qu'ils continuent leur route après le passage de la porte, et encore moins qu'ils continuent leur route sur un «chemin du Jour » différent, quand bien même il ne s'agirait, comme l'a proposé Diels, que d'un chemin à l'intérieur de la citadelle allant de la porte au palais ${ }^{75}$, qui resterait de toute façon bien en deçà de la charge symbolique que les tenants de l'hypothèse de la lumière accordent au « chemin du Jour ».

À nouveau, Hermann Fränkel représente bien la réaction des défenseurs de l'hypothèse de la nuit à cette analyse, dans ses Parmenidesstudien, œuvre fondamentale pour cette hypothèse. Il voit dans la « porte des trajets de la Nuit et du Jour » d'abord la preuve du soi-disant passage du chemin de la Nuit ici-bas au chemin du Jour dans l'au-delà ${ }^{76}$, puis admet dans une note de bas de page insérée ultérieurement qu'il n'y a plus de chemin au-delà de la porte ${ }^{77}$, pour finalement considérer cette contradiction éclatante non pas, comme on s'y attendrait dans une réflexion méthodique, comme une réfutation définitive de sa thèse initiale, mais comme une preuve supplémentaire à l'appui de son opinion déjà établie selon laquelle Parménide, dès que l'on « donne de la valeur à ses images », devient « complètement obscur et confus $\gg^{78}$.

En vérité, ce que l'on trouve dans le texte de Parménide mène à une conclusion tout autre qui discrédite beaucoup moins son talent poétique. Nous pouvons présenter les résultats obtenus jusqu'ici :

- le narrateur est arrivé seulement avec son attelage à un chemin qui est appelé «chemin du dieu », et sur lequel il est conduit par les filles d'Hélios ;

- ce chemin est alors à identifier avec celui que le dieu Soleil parcourt habituellement pendant la journée ;

75. Ibid. p. 50-51.

76. Fränkel 1930, p. 157 : «Ainsi la même chose, la montée de la nuit jusqu’à la lumière, va jusqu'à être représentée quatre fois en trois vers (9-11). D'abord dans le nom de la force qui guide cette ascension, les filles d'Hélios; ensuite dans le lieu quitté, la demeure de la Nuit; puis dans le fait de relever le voile qui couvrait auparavant la tête des jeunes filles; et enfin dans la porte passée, qui sépare le sentier du Jour de celui de la Nuit. »; voir aussi Tarán 1965, p. 13-14: « La porte sépare donc le chemin de la Nuit de celui du Jour. »

77. Fränkel 1960, p. 161, n. $3:$ : Il peut difficilement dans la réalité y avoir une porte qui s'introduise entre deux sortes de chemin (11); elle se transforme donc aussitôt (21/22) en porte d'entrée de la "demeure" de la déesse. »

78. Ibid. 
- le narrateur parvient par ce chemin céleste jusqu’à une « porte des trajets de la Nuit et du Jour », dont la traversée marque la fin du voyage ;

- les « chemins du Jour »du moins sont manifestement à rattacher au chemin céleste du Soleil que le narrateur utilise dans notre monde (lumière du jour = lumière du soleil) ;

- au-delà de la porte, le chemin parcouru jusque-là par le narrateur ne va pas plus loin et ne peut laisser penser à un autre chemin cosmique du même genre, que la Nuit utiliserait pour ses propres trajets.

Il s'ensuit que la Nuit comme le Jour effectuent leurs trajets quotidiens d'est en ouest sur le chemin céleste dont le point de départ est la porte qui, pour cette raison, est appelée « porte des trajets de la Nuit et du Jour ». Le narrateur parcourt ce chemin dans l'autre sens, c'est-à-dire d'ouest en est jusqu'à la porte.

Ce résultat s'accorde parfaitement avec la représentation grecque traditionnelle, selon laquelle la Nuit et son attelage parcourent après le coucher du soleil exactement le même chemin d'est en ouest que le char du Soleil effectue en journée ${ }^{79}$. La « porte des trajets de la Nuit et du Jour » est alors plus précisément la porte par laquelle la déesse de la Nuit et le dieu $\mathrm{du}$ Soleil sortent dans notre monde en alternance régulière pour parcourir vers l'ouest le même chemin qu'ils utilisent l'un à la suite de l'autre. Elle se trouve alors à l'extrémité orientale du chemin céleste et ainsi à la limite de la terre conçue comme disque dans la tradition poétique ${ }^{80}$, là où les bords du disque terrestre et de la coupole éthérée du ciel qui le surplombe se rencontrent ${ }^{81}$. Cette définition permet directement de résoudre un problème pour lequel $\mathrm{H}$. Fränkel, le principal tenant de l'hypothèse de la lumière, s'est donné bien du mal, inutilement semble-t-il, dans le chapitre II des Parmenidesstudien: pourquoi la porte à laquelle le narrateur arrive est-elle gardée précisément par la justice personnifiée $(D i k e)^{82}$ ? Ce n'est que

79. Burkert 1969, p. 10 (=9): « ... il n'est pas vraiment besoin de rappeler les attelages de la Nuit et du Jour sur la façade est du Parthénon. »

80. Il n'y a pas lieu de retirer au récit parménidien son droit poétique d'utiliser la représentation traditionnelle d'un disque terrestre et de postuler avec Mansfeld 1964, p. 245, que la cosmologie décrite dans la doxa, qui suppose une terre sphérique, soit déjà postulée dans le proème.

81. Le fait manifeste d'une part que la porte donne sur l'air céleste (éther) ou plutôt en est remplie (v. 13), d'autre part qu'elle soit rattachée à la Terre par son seuil de pierre (v. 12), permet de placer cette frontière.

82. Sur cette question, voir Fränkel 1930, p. 158-169 (= Fränkel 1960, p. 162-173). Son analyse (ibid. p. 169) est que Dike signifie chez Parménide «la cohérence interne [...] qui permet de postuler les propriétés de l'être à partir de son essence, et même de réguler cellesci. Elle maintient l'être dans sa propre essence et dans sa pureté, elle ne le laisse pas changer ni corrompre sa propre nature. Elle donne ou refuse la connaissance au mortel selon l'es- 
lorsque l'on a reconnu la fonction principale de la porte, à savoir qu'elle permet au Soleil et à la Nuit de passer en alternance stricte et d'entamer leur voyage dans notre monde sur l'unique chemin vers l'ouest, que l'on comprend pourquoi la «justice pleine d'amendes » a la charge de surveiller la porte et par là de décider qui elle va laisser passer et quand. En effet, puisque le Jour comme la Nuit utilisent le même chemin céleste, il faut forcément supposer une succession régulée par une instance judiciaire $^{83}$; chaque jour est amendé par une nuit et cela vaut aussi au niveau de l'année : «L'injustice que le Jour fait à la Nuit pendant l'été doit être réparée en hiver, en quelque sorte au centime près $\gg^{84}$; l'alternance régulée entre le jour et la nuit sert d'exemple cosmique à l'action de Dike. C'est dans la mesure où le narrateur veut passer la porte que passent (dans l'autre sens) quotidiennement le Jour et la Nuit qu'il est sous la responsabilité de Dike.

\subsection{Se décider entre l'hypothèse de la lumière et celle de la nuit}

L'examen des questions $\mathrm{A}$ à $\mathrm{D}$ à partir du texte du voyage a montré que les conditions nécessaires et suffisantes définies en 2.3 pour admettre les deux variantes de l'hypothèse de la lumière n'ont à chaque fois pas été remplies, et que celles pour admettre l'hypothèse de la nuit l'ont été. Nous avons aussi montré que cette dernière propose une reconstruction du voyage cohérente, sans ruptures ni contradictions internes. Celui-ci peut tout à fait être décrit comme un trajet dans le ciel, au sens où le narrateur parcourt le chemin du dieu Soleil auquel ses cavales l'ont conduit; mais la destination vers laquelle les filles d'Hélios le mènent n'est pas la lumière, mais la citadelle de la Nuit, située à l'extrémité orientale du chemin et défendue par la porte des trajets du Jour et de la Nuit.

\subsection{Qui est la déesse de la révélation?}

Une fois l'hypothèse de la nuit démontrée, l'identité de la déesse de la révélation n’est plus douteuse : il s'agit, comme Morrison le suppose, de la

sence que lui a donnée Moira. » On trouve la même chose en plus simple mais guère plus éclairant chez Deichgräber 1958, p. 664 (cité supra n. 37).

83. Morrison 1955, p. 60 : «En tant que gardienne des clefs de la Nuit et du Jour, elle [Dikè] préside à l'ordre quotidien et exerce un principe régulateur de justice pour ajuster les relations de l'un avec l'autre. C'est ainsi qu'Anaximandre concevait que l'ordre du monde était régulé (DK 12 B 1), et Héraclite exprimait une idée semblable quand il disait que le Soleil "n'outrepassera pas son rôle; sinon, les Érinyes, servantes de Dikè, le trouveront" (DK 22 B 94) ». Il faut aujourd'hui comparer la citation héraclitéenne de Morrison avec la col. IV du papyrus de Derveni, où le fragment $\mathrm{B} 94$ est directement précédé d'une version du fragment B 3 ; voir Kouremenos/Parássoglou/Tsantsanoglou 2006, p. 69 et 154-161.

84. Burkert 1969, p. 10-11 (=9-10): «Das Unrecht, das der Tag im Sommer der Nacht antut, muß er ibr im Winter zurückzablen, gleichsam auf Heller und Pfennig ». 
maîtresse de la forteresse sur laquelle donne la porte, c'est-à-dire la déesse de la Nuit ${ }^{85}$. Walter Burkert, qui a d'abord laissé la question ouverte (au motif que la terminologie des mystères est volontairement vague sur ce sujet $)^{86}$, montre dans un « addendum 2007 » que le papyrus de Derveni, édité officiellement en 2006, a confirmé que la déesse de la Nuit avait déjà dans la poésie orphique un rôle de conseiller cosmogonique ${ }^{87}$.

Certes, Kingsley (1999) a soulevé une objection contre une assimilation entre la déesse de la révélation et la Nuit : la déesse, si elle était la Nuit, ne saurait alors parler avec justesse du Feu et de la Nuit comme des deux principes établis par les mortels ${ }^{88}$. Mais en conclusion de cette étude, nous montrerons en comparant le récit du voyage et la révélation que ce que Kingsley considérait comme une faiblesse est en réalité une force : de même que la déesse oppose le pur « est » à l'alternance illusoire entre être et nonêtre, de même le narrateur, en passant la porte, laisse derrière lui l'apparente alternance entre le jour et la nuit pour atteindre la citadelle de la Nuit.

Les alternatives envisagées par quelques tenants de l'hypothèse de la nuit ne peuvent convaincre. L'hypothèse de Sextus Empiricus, qui identifie la maîtresse de la citadelle à la gardienne Dikè ${ }^{89}$, est, comme Burkert (1969) l'a déjà établi, à rejeter comme paradoxe sociologique ${ }^{90}$. Mais les hypothèses selon lesquelles le voyage va beaucoup plus loin après le passage de la porte, que ce soit jusqu'à Mnémosyne, en s'appuyant sur les Lamelles d'or orphiques $^{91}$, ou jusqu'à Perséphone elle-même, comme dans le mythe de la catabase $^{92}$, sont incompatibles avec le texte du voyage: elles remplacent le voyage dont il est dit dans le texte qu'il mène au point de départ des filles d'Hélios, clairement désigné comme la citadelle de la Nuit située juste derrière la porte, par un voyage plus lointain dont on ne trouve mot dans le texte et qui mènerait à une destination dont on ne trouve pas davantage mot dans le texte.

85. Morrison 1955, p. 60 : «elle vit dans la "demeure de la Nuit", et est donc probablement la Nuit elle-même. »

86. Burkert 1969, p. 13-14 (=12).

87. Burkert 2008, p. 26 ; Palmer 2009, p. 59-61. Voir plus loin section 4.5.

88. Kingsley 1999 , p. 93 « [...] plus tard dans le poème, quand elle en arrive à parler de la Nuit et du Jour, elle dit que ce sont deux contraires illusoires dans un monde trompeur. Ce n'est pas une manière de parler de soi-même. »

89. Mansfeld 1964, p. 261-272, va dans le même sens.

90. Burkert 1969, p. 13 (=11-12) : « Le gardien de porte et le maître de maison sont dans la vie quotidienne des Grecs naturellement séparés : le gardien laisse entrer, le maître ou la maîtresse de maison accueille. »

91. Sassi 1988, p. 394-395.

92. Kingsley 1999, p. 94 : «Parménide est descendu aux enfers jusqu’à une déesse qui vit dans le royaume de la mort. Les Grecs l'appellent Perséphone. » Voir les objections très justement soulevées par Palmer 2009, p. 58-59. 
4. Le récit de voyage parménidien et la tradition poétique : Hésiode, Mimnerme, Stésichore, Orphée

\subsection{Introduction}

L'argumentation que nous avons menée jusqu'ici, en raison de l'état de la recherche esquissé en introduction, ne s'est appuyée que sur le texte de Parménide. Cela ne signifie cependant pas que nous doutions du fait, bien établi par Morrison et Burkert, que Parménide puise de multiples manières dans la tradition poétique. Au contraire : un rapide regard sur la poésie qui précède Parménide peut montrer combien la lecture du récit de voyage selon l'hypothèse de la nuit et particulièrement l'initiation du narrateur par la déesse de la Nuit a dû sembler limpide à un auditoire pré-platonicien, qui n'était pas encore conditionné par l'allégorie de la caverne de la République à assimiler connaissance et chemin vers la lumière. Insistons à nouveau, par mesure de précaution, sur le fait que la sélection de textes que nous allons proposer sert à illustrer et préciser une interprétation déjà fondée, et non à justifier notre choix fondamental de l'hypothèse de la nuit contre celle de la lumière.

\section{2 Hésiode, Théogonie, 746-754}

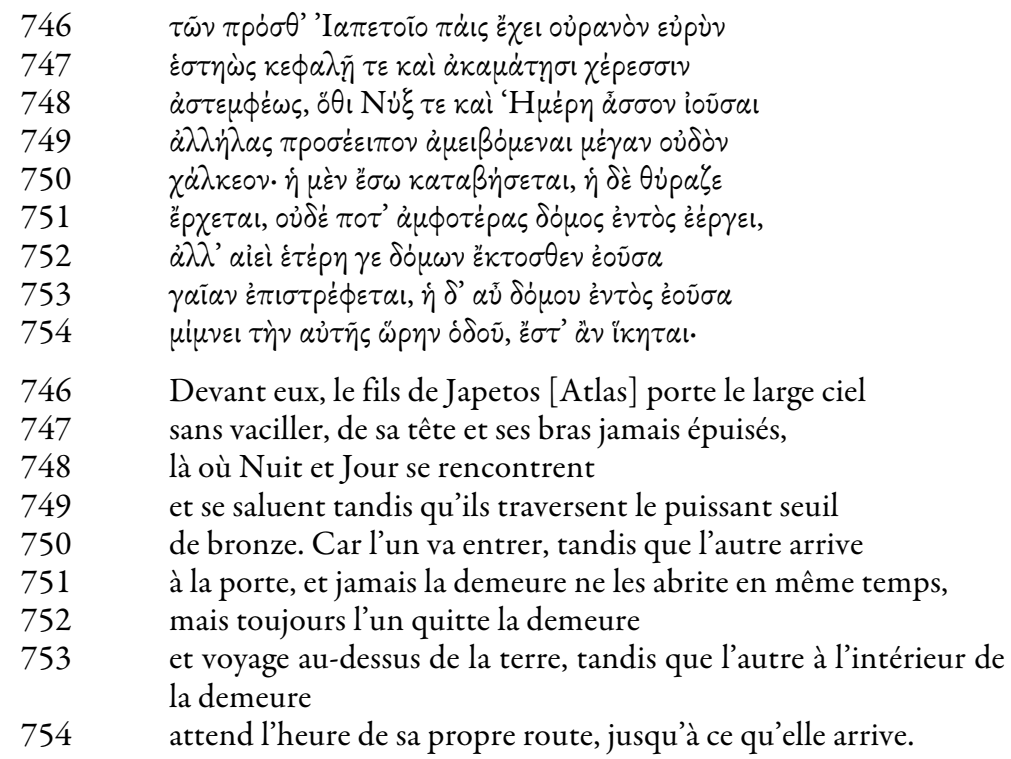

À la limite du disque terrestre, au bord de l'abîme qui mène à la prison des Titans, se trouve la demeure qui abrite en alternance le Jour et la Nuit et qui, comme une interpolation rhapsodique le montre, a été identifiée 
dans la tradition hésiodique comme « demeure de la Nuit ${ }^{93}$. C'est là que le Jour se repose pendant que la Nuit traverse la terre et inversement: quand l'un rentre, l'autre s'en va. Par ailleurs, la demeure, comme le montre la mention d'Atlas, est située à l'ouest du disque terrestre, et donc là où à chaque fois la Nuit et le Jour se couchent à la fin de leur trajet. Mais Hésiode n'explique pas comment ils peuvent reprendre à l'est leur parcours après leur repos respectif ${ }^{94}$.

4.3 Mimnerme, fr. 12 West (2 moitié du VII siècle av. J.-C.)

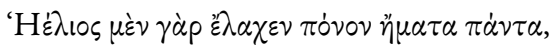

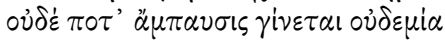

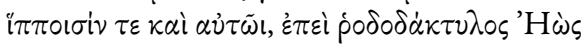

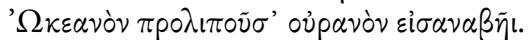

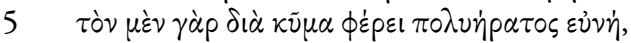

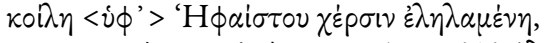

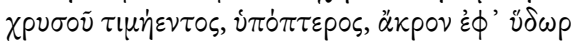

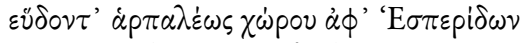

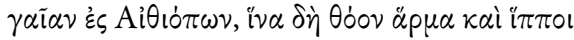

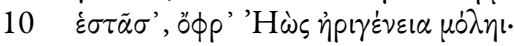

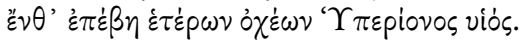

Le lot d'Hélios est de travailler tous les jours ; jamais aucune pause n'est impartie à ses cavales ni à lui à partir du moment où Aurore aux doigts de rose, quittant l'Océan, est montée dans le ciel.

5 Car sa couche très enviée le porte à travers les flots, (elle est forgée en creux des mains d'Héphaïstos à partir d'or précieux, avec des ailes) à la surface de l'eau, alors qu'il dort, et va rapidement du domaine des Hespérides [de l'ouest] jusqu'à la terre des Éthiopiens [à l'est], où le char rapide et ses cavales 10 attendent qu'Aurore née de bonne heure arrive. Là, le fils d'Hypérion [c'est-à-dire le Soleil] monte sur son nouvel attelage.

Mimnerme répond à la question qui restait ouverte chez Hésiode, du moins pour le jour : le Soleil revient après son coucher à l'ouest (domaine des Hespérides) vers son point de départ à l'est en étant reconduit pendant la nuit vers l'est (terre des Éthiopiens) dans un récipient d'or qui lui sert d'embarcation sur le fleuve Océan, qui coule autour du disque terrestre. Si ce voyage sur l'Océan n'est pas associé à un repos dans la demeure de la

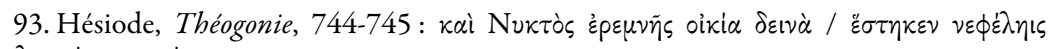

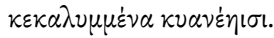

94. Voir West 1966, p. 366-367, pour la variante $\dot{\alpha} \mu \phi i \varsigma$ દ̇oṽ $\sigma \alpha \mathrm{l}$ au vers 748. 
Nuit (que ce soit à l'ouest avant de partir ou à l'est après son voyage), c'est à cause du propos spécifique de cette élégie : elle doit souligner que la vie du Soleil n'est que travail et que le gîte qu'il désire subjectivement n'est objectivement qu'un moyen de le transporter à l'endroit d'où il doit repartir le matin suivant au travail.

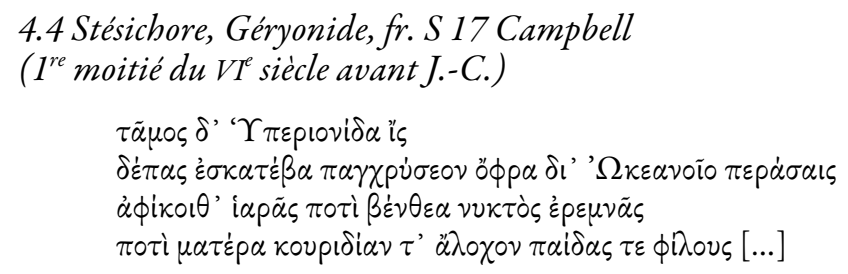

Et alors le vigoureux fils d'Hypérion [c'est-à-dire le Soleil] monta dans sa coupe d'or pur pour traverser l'Océan et ainsi atteindre, dans les profondeurs de la nuit sacrée et obscure, sa mère, son épouse légitime et ses chers enfants [...]

Stésichore décrit le coucher du Soleil. Le dieu du Soleil Hélios, arrivé au bout de son chemin à l'ouest, monte dans le récipient d'or déjà introduit par Mimnerme et traverse ainsi l'Océan qui coule autour du disque terrestre ; depuis l'autre rive de l'Océan il atteint alors les profondeurs de la nuit. Comme le dieu n'utilise pas le fleuve circulaire comme chez Mimnerme pour aller vers la limite orientale du disque terrestre, mais ne fait que le traverser ${ }^{95}$, la demeure de la Nuit devrait chez Stésichore comme déjà chez Hésiode se trouver à l'extrémité ouest. Bien que le fragment conservé ne l'affirme pas, on peut imaginer que comme chez Mimnerme, Hélios retourne après s'être reposé vers son point de départ à l'est, en empruntant son récipient d'or sur l'Océan. Par rapport à Parménide, il faut particulièrement noter que Hélios retrouve dans les profondeurs de la nuit sa mère, sa femme et ses enfants; parmi ses enfants se trouvent en effet le malheureux Phaéton, mais aussi ses sœurs, les filles d'Hélios, qui jouent un rôle décisif dans le voyage de Parménide.

\subsection{Orphicorum Testimonia et Fragmenta 237 F, 1-3 Bernabé}

$\mu \alpha \tilde{\alpha} \alpha, \theta \varepsilon \tilde{\omega} v \dot{v} \pi \dot{\alpha} \tau \eta, N i \dot{\xi} \ddot{\alpha} \mu \beta p o \tau \varepsilon, \pi \tilde{\omega} \zeta, \tau \dot{\alpha} \delta \varepsilon \phi \rho \dot{\alpha} \zeta \varepsilon$,

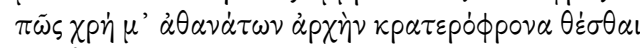

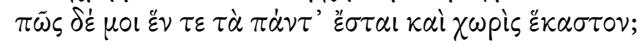

Ma mère, suprême parmi les dieux, Nuit immortelle, comment, dis-le moi, comment dois-je établir mon ferme pouvoir sur les immortels, comment est-ce que tout sera un pour moi et [puis à nouveau] séparé l'un de l'autre?

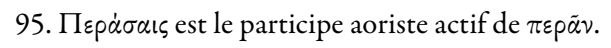


Dans ces vers tirés d'une théogonie orphique, qui sont cités au plus tôt par Proclus et remontent donc sous cette forme peut-être à la version impériale et «rhapsodique » de cette théogonie, Zeus va chercher conseil auprès de la Nuit, « suprême parmi les dieux », pour apprendre comment il peut exercer le pouvoir sur les autres immortels. Comme ce témoignage est tardif, seule la position initiale de la Nuit dans le processus de la théogonie, garantie par le témoignage du disciple d'Aristote Eudème, a longtemps pu être considérée comme un motif orphique ancien ${ }^{96}$. Cela a changé avec la découverte (1962) et surtout l'édition (2006) du papyrus de Derveni (IV ${ }^{\mathrm{e}}$ siècle avant J.-C.) : dans la théogonie orphique qui y est citée et interprétée allégoriquement, la Nuit fait office de conseiller cosmogonique auprès de Zeus ${ }^{97}$; ce motif peut donc aujourd'hui être considéré comme appartenant à l'orphisme ancien ${ }^{98}$. Cela livre un contexte contemporain possible à l'apparition de la déesse de la Nuit pour révéler l' $\alpha \lambda \dot{\eta} \theta \varepsilon ı \alpha$ et la $\delta$ ó $\xi \alpha$ que l'on trouve chez Parménide.

\subsection{Conclusion : tradition et innovation dans le récit de voyage}

Si l'on admet l'interprétation démontrée auparavant, qui s'accorde avec l'hypothèse de la nuit, les quatre passages que nous avons présentés montrent que Parménide n'aligne pas des motifs de la tradition poétique sans les structurer, comme les partisans de l'hypothèse de la lumière doivent l'affirmer. Mais il a modifié ces motifs de manière systématique et les a intégrés dans un nouveau tout. Il part ainsi du motif hésiodique de la demeure où le Jour et la Nuit se reposent tour à tour de leur voyage sur le chemin céleste. Il concrétise ce scénario grâce à la représentation asymétrique particulière à Stésichore, selon laquelle ce lieu de repos à la fin du chemin est le domaine de la Nuit, et ne sert pas seulement, comme on pourrait le comprendre, à la Nuit, mais aussi au Soleil, et où même les filles de celui-ci résident. Parménide présuppose l'idée, claire par elle-même et que l'on trouve chez Mimnerme, selon laquelle le fleuve Océan permet au Soleil et à la Nuit de

96. Orphicorum Testimonia et Fragmenta, 20 F Bernabé

97. Kouremenos/Parássoglou/Tsantsanoglou 2006, p. 183 : « Dans la col. XI, l’auteur du papyrus de Derveni analyse deux hexamètres dans lesquels la Nuit fait des prophéties à quelqu'un depuis un adytum, et dans la col. XII il examine un hexamètre où le pouvoir de quelqu'un sur Olympe est présenté comme une cause finale. À la lumière des Rhapsodies orphiques ultérieures, où la Nuit faisait à Zeus la prophétie qu'il était destiné à renverser son père Cronos et lui donnait des instructions de teneur cosmologique, on peut penser que le futur dirigeant de l'Olympe, col. XII, est le même que celui qui reçoit la prophétie de la Nuit col. XI, que ces prophéties encouragent celui-ci à devenir roi de l'Olympe, et que ce sont les $\theta \dot{\varepsilon} \sigma \phi \alpha \tau \alpha$ que Zeus a entendus avant de renverser son père (col. VIII.4-5). »

98. Ainsi l'«addendum 2007 » de Burkert 2008, p. 20, doit être lu comme une relativisation implicite de ses réserves émises auparavant (1969, p. 13-14 [=12]) contre l'assimilation par Morrison de la déesse de la révélation à la Nuit. 
revenir à l'est depuis le point d'arrivée à l'ouest de leur voyage dans notre monde. Enfin, il introduit l'assertion que le Soleil et la Nuit ne se reposent pas à l'ouest (comme chez Hésiode et Stésichore), c'est-à-dire avant le retour, ni pendant leur trajet (comme chez Mimnerme), mais seulement à l'est, c'est-à-dire après le retour. Cela se tire du fait que l'habitation de la Nuit, où le dieu Soleil et ses filles trouvent leur gîte, est chez Parménide comme chez Stésichore tout près de l'entrée orientale du chemin céleste, à savoir dans la citadelle de la Nuit, au-delà de la porte des trajets de la Nuit et du Jour. En effectuant ce déplacement, Parménide s'est donné la possibilité d'approfondir le motif hésiodique des allées et venues alternées de la Nuit et du Jour par la nouvelle image frappante de la porte par laquelle la déesse de la justice, en tant que gardienne, laisse passer le Soleil et la Nuit, chacun à son tour, pour rejoindre le chemin céleste. Enfin, le primat de la déesse de la Nuit par rapport aux autres dieux et son rôle de conseiller cosmogonique omniscient apparaissent, grâce à Eudème d'un côté, au papyrus de Derveni de l'autre, comme des motifs orphiques anciens. Cet indice positif est complété du côté négatif par la démonstration déjà proposée par Classen (1965) selon laquelle dans les fragments conservés de la philosophie grecque pré-parménidienne, en dépit de la signification cosmologique et zoogonique du feu, il n'y a aucun signe de l'existence d'une « métaphysique de la lumière ${ }^{99}$. Devant cette toile de fond, on doit admettre que notre manière de comprendre le récit de voyage, démontrée à partir du texte de Parménide et qui va dans le sens de l'hypothèse de la nuit, était aussi claire à l'auditeur contemporain du poème de Parménide qu'elle est obscure au lecteur moderne.

\section{Le récit de voyage et la révélation de la déesse}

\subsection{Les deux voies de recherche et leur amalgame par les mortels}

Après que la déesse de la Nuit a salué avec bienveillance le narrateur, elle lui fait une double révélation : d'un côté le cœur inébranlable de la vérité

99. Classen veut ainsi contester l'application de l'affirmation globale de Kranz 1916, p. 1163 («Lumière et vérité, obscurité et mensonge sont identiques aussi pour les Grecs depuis toujours ») au domaine de la philosophie pré-parménidienne, tandis qu'il lit le voyage de Parménide lui-même dans le sens de l'hypothèse de la lumière. En introduisant l'hypothèse de la nuit, cependant, on voit que le discours parménidien sur l'aletheia se serait glissé sans problème dans la perspective générale développée par Classen, et que la nouveauté parménidienne qu'il décrit se limiterait au discours sur la doxa; voir B 8, 56 - -59 : $\tau \tilde{\eta} \iota \mu \varepsilon \dot{\nu}$

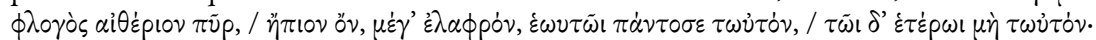

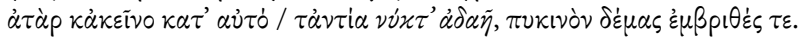


entièrement convaincante ${ }^{100}$, de l'autre les opinions (doxai) des mortels dans lesquelles ne réside aucune vraie force de conviction ${ }^{101}$.

La partie sur la vérité concerne les deux voies de recherche ${ }^{102}$, qui seraient les seules qu'il y ait à penser. Chacune de ces deux voies est esquissée par deux phrases. À chaque fois, la première phrase n'est composée que d'un prédicat incomplet. Pour la première voie, ce prédicat est: « est »; pour la seconde au contraire, le prédicat est négatif: «n'est pas $»^{103}$. Après ces prédicats, on trouve à chaque fois une phrase qui contient une détermination modale du non-être (impossible/nécessaire) ${ }^{104}$ :

$$
\begin{aligned}
& 1^{\text {re }} \text { voie de recherche : « est et il n'est pas possible de ne pas être. » } \\
& 2^{\text {nde }} \text { voie de recherche }: \text { « n'est pas et il est nécessaire de ne pas être. » }
\end{aligned}
$$

L'introduction de cette détermination modale rend évident que les deux voies tiennent des propos opposés (impossible/nécessaire) sur la même chose (le non-être). Elles sont donc incompatibles : qui choisit la première voie doit rejeter la seconde, et qui choisit la seconde voie doit rejeter la première. On a ici un «ou » exclusif très net ${ }^{105}$; à partir de là, il ne peut en aucun cas y avoir de lien entre un être et le « n'est pas ${ }^{106}:$ : rien n'estpas ».

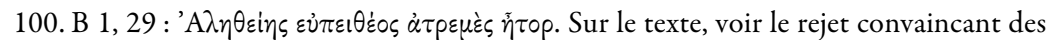

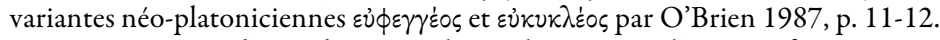

101. En guise d'introduction à la révélation, voir l'instructif essai critique d'O'Brien 1987, p. 135-302. Pour un traitement détaillé des controverses interprétatives qui se présentent à tous les tournants, on peut renvoyer à Wiesner 1996, qui a cependant réservé l'examen du long fragment $\mathrm{B} 8$ à une publication ultérieure.

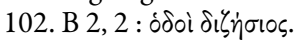

103. On a fait diverses propositions pour compléter les prédicats sans sujet « est » et « n'est pas » avec un sujet sous-entendu ; le complément choisi dépend alors naturellement de l'interprétation que chacun a du verbe «être » (voir là-dessus O’Brien 1987, p. 157169 et Wiesner 1996, p. 205-228). Un tel procédé se trouve par exemple quand quelqu'un suppose comme Mourelatos 1976, p. 59, que « l'opposition entre être et non-être est celle entre détermination et indétermination », et complète alors la traduction de la description des deux voies comme Schmitt 2007, p. 114 : « une voie selon laquelle <quelque chose de déterminé $>$ est, et il n'est pas possible que rien $<$ de déterminé $>$ ne soit $[\ldots]$ une autre selon laquelle quelque chose de non <déterminé> est <possible $>$, et même un être non $<$ déterminé> est nécessaire. » ( «Der eine Weg, dass <Bestimmtes $>$ ist, und dass es nicht möglich ist, nichts <Bestimmtes $>z u$ sein... Der andere, das Nicht $<-$ Bestimmtes $\rangle\langle$ möglich $>$ ist, ja dass Nicht <Bestimmt-> Sein notwendig ist.») Mais indépendamment de la valeur de l'explication qu'ils présentent pour le prédicat que nous avons (《est »), par exemple ici « est quelque chose de déterminé », la phrase «il/elle/c'est quelque chose de déterminé » n'a pas le même sens que la phrase « quelque chose de déterminé est ».

104. B 2, 3-8.

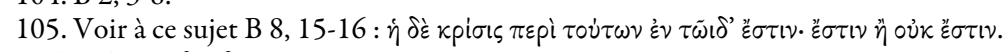

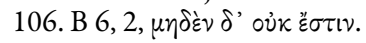


Les deux voies de recherche sont évaluées par la déesse comme suit : la première est la voie de la vérité et de la conviction; elle permet de comprendre (B 8, 3-6) que l'être est inengendré (à $\left.\gamma^{\varepsilon} v \eta \tau o v\right)$ et incorruptible

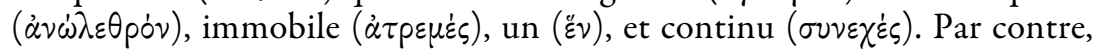
la seconde voie est absolument impraticable car nous ne pouvons connaître ni dire « ce qu'il n'y a pas ${ }^{107}$ : ce n'est pas une vraie voie ${ }^{108}$. La voie du « est » mène à une explication de l'«être », tandis que la voie du «n'est pas » ne conduit nulle part : elle n'est pas juste un détour ou une fausse route, mais une non-route.

Quel est le rapport entre la doxa et les deux voies de recherche présentées dans l'aletheia ? Les opinions des mortels, que Parménide développe et dont il montre les fondements dans la doxa, ne se laissent facilement comparer à aucune des deux voies de recherche: pas avec la première, puisqu'il n'y aurait alors plus d'opposition entre aletheia et doxa, ni avec la seconde, puisqu'elle est indicible et impensable, une hypothèse de pensée purement fictive, que personne ne peut réellement adopter, et encore moins le commun des mortels.

Karl Reinhardt a tiré du fragment B 6 l'hypothèse convaincante que Parménide présente en fait dans la doxa des mortels une troisième voie ${ }^{109}$ : dans ce fragment en effet, après avoir reconnu la première voie de recherche, Parménide rejette deux voies, à savoir d'abord la seconde voie de recherche, et ensuite une autre voie, non mentionnée jusque là, qui diffère des deux précédentes : il s'agit de la voie où titubent les mortels ignorants à double tête ${ }^{110}$. Dans son contenu, la voie des mortels se définit comme un va-et-vient obscur entre l'amalgame et la séparation des deux voies de recherche, c'est-à-dire l'« est » et le «n'est-pas » : tantôt ils considèrent qu'être et non-être sont équivalents, tantôt non ${ }^{111}$. Cette oscillation se montre erronée vis-à-vis de l'incompatibilité présentée en introduction par la déesse entre « est » et «n'est pas ». La description méprisante des mortels comme «à double tête » (B 6, 5 dikranoi) pointe vers cette versatilité caractéristique de leur opinion par rapport aux deux voies de recherche. En

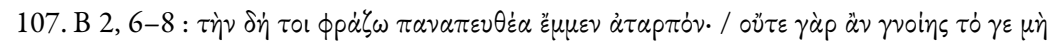

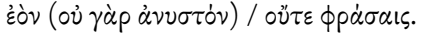

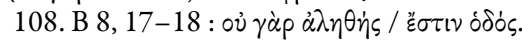

109. Reinhardt 1916, p. 35-36. Voir Mansfeld 1964, p. 1-41.

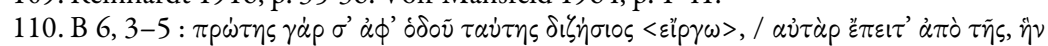

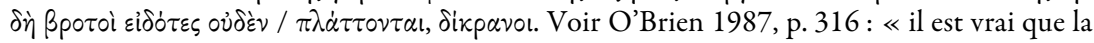
déesse donne de la valeur à cette erreur en l'appelant “voie" (fr. $7.2 ; c f$. fr. 6.4). Mais ce n'est une "voie" que dans le sens où c'est un chemin foulé par des mortels qui ne sont pas parvenus à voir qu'il n'y a à proprement parler que deux "voies" pensables : "est" et "n'est pas" (fr. 2). »

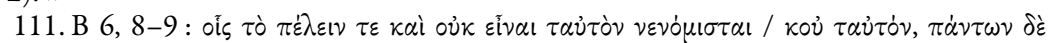

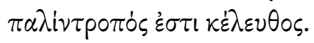


particulier, l'amalgame entre « est » et « n'est-pas » se remarque dans le fait qu'ils sont associés dans une seule et même phrase, par exemple dans celle qui est rejetée comme anarchique en $\mathrm{B} 7,1:$ : il y a un non-être ${ }^{112}$. Pour ne pas tomber dans la troisième voie, il faut faire taire le témoignage de la perception sensible pour juger seulement par la raison la démonstration pugnace de la déesse ${ }^{113}$.

C'est précisément la raison pour laquelle il faut expliquer pourquoi la déesse propose également d'offrir au narrateur une présentation détaillée des opinions des mortels (doxai). Pour répondre à cette question, nous nous tournons à nouveau vers le voyage raconté dans le proème - interprété dans le sens de l'hypothèse de la nuit ${ }^{14}$.

\subsection{La structure ternaire dans le voyage et l'aletheia ${ }^{115}$}

Le récit de voyage repose sur une structure ternaire où un élément apparaît deux fois, un autre une seule : la nuit et le jour alternent devant la porte ; derrière la porte, il n'y a que la Nuit, pas de jour, et cela même quand le Soleil se repose dans la citadelle de la Nuit : il ne fait naturellement jour que pendant la journée. Le narrateur voit alors l'alternance du Jour et de la Nuit devant la porte, tandis qu'il reçoit la révélation de la déesse de la Nuit derrière celle-ci :

\section{Nuit}

\section{Jour}

Nuit

L'aletheia repose aussi sur une telle structure ternaire : on peut se représenter deux voies de recherche, « est » et «n'est pas »; et dans le monde du changement, du devenir et de la corruption tel qu'il se présente aux sens des mortels, « est » et «n'est pas » coexistent et alternent. Mais dans la vérité, la voie du «n'est pas » est impraticable, c'est pourquoi une explication du monde qui repose sur l'oscillation entre «est » et «n'est pas » ne peut absolument pas tenir devant la raison; celle-ci ne peut de fait accepter que la voie du « est »:

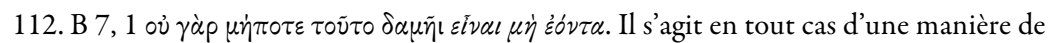
parler que Parménide rejette, que l'on traduise o $\nu^{\prime} \dot{\eta}^{\prime} \pi \circ \tau \varepsilon \delta \alpha \mu \tilde{\eta} \iota$ strictement par « ne sera jamais vaincu [c'est-à-dire domestiqué, adopté, intégré] » (voir O’Brien 1987, p. 46) ou plus librement par «ne sera jamais forcé » (Kranz, Wiesner).

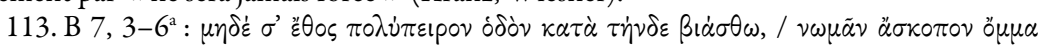

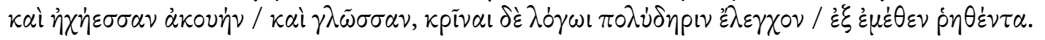

114. En dépit du verdict prononcé par Barnes 1982, p. 156: «Le poème commençait par un long prologue allégorique dont l'interprétation est dans l'ensemble de peu d'importance d'un point de vue philosophique. »

115. À propos de la thèse développée par la suite, voir aussi Primavesi 2005, p. 76-82. 


$$
\begin{aligned}
& \ll \text { est } \gg \\
& \text { «n'est pas » }
\end{aligned}
$$

À l'encontre de l'objection soulevée par Kingsley contre l'assimilation de la déesse et de la Nuit ${ }^{116}$, nous avons montré que l'analogie structurelle entre le récit de voyage et l'aletheia repose précisément sur la double mention de la Nuit : dans notre monde, elle fait partie d'une alternance, dans l'au-delà, elle seule domine.

\subsection{Le voyage et le chemin de la doxa à l'aletheia}

On est autant frappé par la structure parallèle entre voyage et aletheia que par la différence suivante : dans le récit de voyage, la succession du Jour et de la Nuit et la citadelle de la Nuit se trouvent dans le même univers où un voyage, un passage, est réalisé de l'une à l'autre. Ce passage s'exécute en deux phases: de même qu'il est difficile de douter que le but du voyage est la révélation faite par la déesse de la Nuit (c'est le point d'arrivée de la seconde phase), on peut tout aussi peu contester que le narrateur devait d'abord être porté au chemin du dieu par ses cavales, c'est-à-dire par son propre impetus poético-philosophique, pour pouvoir être escorté ensuite par les filles d'Hélios jusqu'à la déesse de la Nuit (c'est le point d'arrivée de la première phase). Il était donc déjà parvenu bien au-delà du monde quotidien des mortels, à la surface de la terre, pour atteindre le chemin céleste et avait ainsi utilisé dans une mesure non négligeable ses propres forces avant que les filles d'Hélios ne prennent son parti et n'apportent une aide divine à l'audace du héros philosophique, comme la déesse de la Nuit le fera ensuite. La relation entre les hauts faits du narrateur parménidien lui-même et l'aide divine complémentaire qui lui est ensuite accordée correspond à un principe qui est énoncé à plusieurs reprises dans la tragédie contemporaine d'Eschyle : « la divinité soutient volontiers celui qui se donne de la peine ${ }^{117}$. »

Dans l'aletheia au contraire, la déesse approuve expressément la voie du « est » et nie juste abruptement la voie du «n'est pas » ainsi que l'amalgame entre les deux : elle n'évoque pas le passage de la doxa à l'aletheia, de l'illusion d'une alternance à la reconnaissance de l'unité ; mais au contraire l'aletheia, en distinguant les deux voies, fournit la condition absolue pour expliquer l'existence du monde trompeur de la perception tel qu'il est décrit dans la doxa.

116. Kingsley 1999, p. 93.

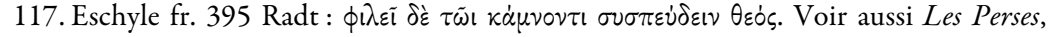

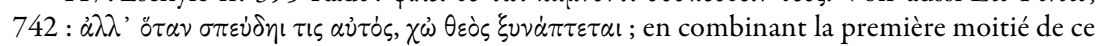
vers avec la fin de vers aristophanienne $\chi \dot{\omega} \theta \varepsilon \dot{s} \xi \xi u \lambda \lambda \dot{\eta} \psi \varepsilon \tau \alpha \mathrm{l}$ (Les Cavaliers, 229b), U. von Wilamowitz-Moellendorff a construit le vers qui conclut le prélude à son anthologie grec-

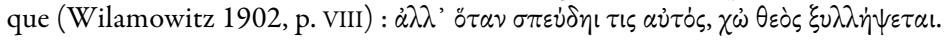


Mais si on essaye de regarder la physique de la doxa non pas du point de vue désobligeant de la déesse, mais de celui du narrateur humain, on remarque que la déesse ne présente pas dans la doxa un amoncellement d'opinions que les hommes ont au jour le jour, ce qui correspondrait au point de départ terrestre du voyage, mais qu'elle remonte aux principes de cette pensée du quotidien. Ces principes sont deux : le Feu et la Nuit ${ }^{118}$, étant donné que toutes les apparences du monde de la doxa reviennent à ces deux principes ${ }^{119}$. Ceux-ci correspondent d'une manière qui peut difficilement être une coïncidence au Jour (et en particulier au Soleil) et à la Nuit dont le chemin céleste est atteint par le narrateur au terme de la première étape de son voyage ${ }^{120}$. Si l'on pense jusqu'au bout cette analogie, la partie de la révélation concernant la doxa se conçoit ainsi comme un chemin vers l'aletheia qu'un mortel ne peut omettre, de même que le voyage vers le « chemin du dieu » est un chemin vers la révélation de la déesse de la Nuit et ne peut être omis par un mortel. Par conséquent, la doxa renvoie au but de la première phase du voyage, à savoir atteindre le chemin céleste du Soleil et de la Nuit.

Cette position intermédiaire de la doxa est dissimulée dans la révélation par le fait que la déesse, pour parler comme Aristote ${ }^{121}$, va du « plus connu en soi » (aletheia) au «plus connu pour nous » (doxa) jusqu'à un certain point : la via expositionis dépend de son autorité divine en ce qu'elle commence par l'être et non par les opinions confuses, et n'entre dans le monde des opinions (selon les fragments conservés) que dans la mesure où elle ramène leur caractère changeant aux deux principes immanents. En revanche, le voyage a une fonction complémentaire en ce qu'il montre le chemin pour les humains qui va dans l'autre sens, du «plus connu pour nous » $($ doxa $)$ au «plus connu en soi » (aletheia), autrement dit en passant par une via inventionis qui conduit d'abord des opinions confuses,

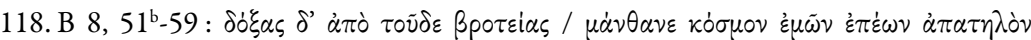

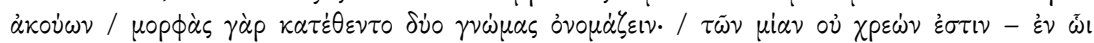

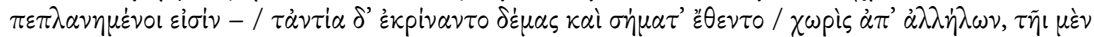

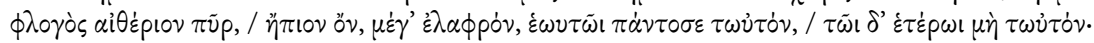

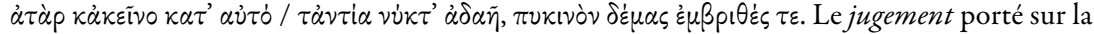
lumière et la nuit dans les vers 57 et 59 correspond naturellement aux doxai des mortels et non au point de vue supérieur de la déesse, contrairement à ce qui se passe en B 14-15, comme nous le verrons par la suite.

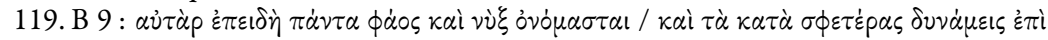

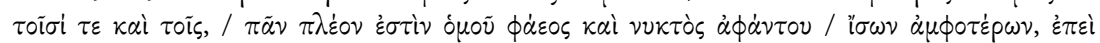

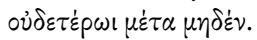

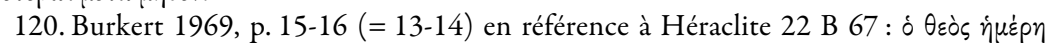

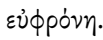

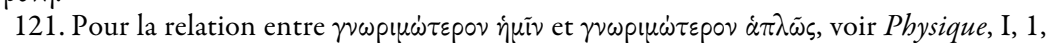
184a15-16; Métaphysique, Z, 3, 1029b3-12 ; Éthique à Nicomaque, I, 4, 1095b2-4. 
grâce à l'impetus poétique et noétique du narrateur, aux deux principes de la doxa, puis, grâce à l'escorte divine, jusqu'à la révélation de l'être. Malgré l'écart considérable qui existe dans la forme et le fond entre le poème parménidien et les Analytiques postérieurs d'Aristote, la relation entre le voyage illustré narrativement et la révélation de la déesse est semblable à celle entre le chemin vers les principes d'Aristote et son apodictique.

\subsection{Critique de la lumiere lunaire ${ }^{122}$}

Il reste à expliquer le sens philosophique qu'il y a à attribuer la révélation à la déesse de la Nuit. Pour illustrer narrativement le chemin de la connaissance depuis la doxa binaire jusqu'à l'aletheia moniste, on pouvait en soi, si l'on fait abstraction des formulations parménidiennes analysées dans la section 3, concevoir un voyage qui décrive un passage depuis l'alternance entre le Jour et la Nuit jusqu'au royaume de la lumière éternelle. On peut enfin comprendre pourquoi Parménide conduit son narrateur jusqu'au royaume de la Nuit dans l'au-delà si l'on remarque que la lumière du jour est la condition nécessaire pour voir et ainsi pour la perception sensible par excellence : l'alternance entre la nuit et la lumière du jour illustre le monde binaire de l'opinion des mortels dans la mesure où en ce bas monde la perception sensible est considérée comme positive et certaine, et son absence ou son impossibilité comme négative et incertaine. En revanche, l'obscurité permanente qui règne dans la citadelle de la Nuit correspond à un lieu où la perception sensible, reconnue comme trompeuse, est par principe impossible. La déesse de la Nuit est donc à juste titre celle qui révèle l'aletheia parce que, dans sa citadelle de l'au-delà, même les «nombreuses différences $\gg^{123}$ suggérées par la vue perdent leur pouvoir ${ }^{124}$.

Si l'on suit Karl Popper ${ }^{125}$, un indice clair de ce renversement du jugement porté sur la lumière et l'obscuritét ${ }^{126}$, et par là du passage à l'aletheia, serait déjà donné par la déesse dans la doxa. Même dans le peu qui nous est conservé de la doxa parménidienne, il a identifié un passage - la définition

122. À ce sujet, voir Sedley 1999, p. 124.

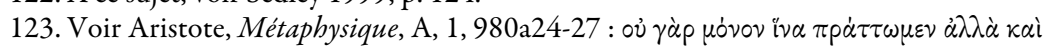

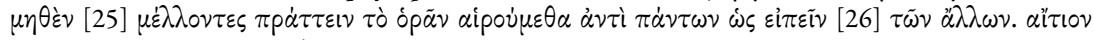

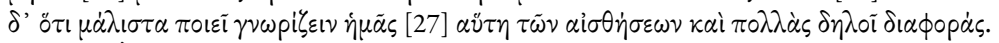

124. À l'occasion de la discussion de la version allemande de cet essai, Gyburg Uhlmann a fait remarquer avec raison que l'élimination des différences dans l'aletheia de Parménide ne concerne que les différences suggérées par la perception sensible, mais qu'elle est de toute évidence compatible avec la distinction conceptuelle entre divers aspects (semata) de l'être.

125. Popper 1992. L'auteur remercie Jaap Mansfeld, à qui Popper a dédicacé son travail, pour avoir remarqué la valeur de cet essai pour l'appréciation philosophique de l'hypothèse de la nuit.

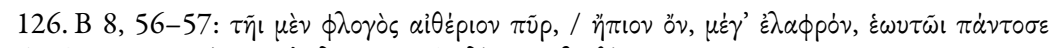

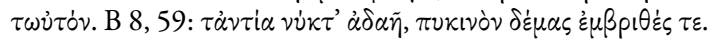


de la lumière de la lune comme simple reflet de la lumière solaire - qui offre un exemple spectaculaire du manque de fiabilité de la perception sensible. Dans le titre paradoxal de son essai ${ }^{127}$, il a en outre manifesté l'intuition que ce passage jette aussi une «lumière sur les deux voies de Parménide ». Popper renvoie à B 14 et B $15^{128}$ :

$$
\begin{aligned}
& \text { Mondgöttin und Sonnengott }{ }^{129}
\end{aligned}
$$

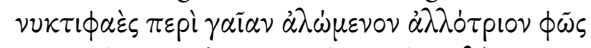

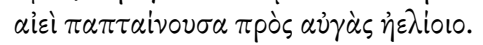

Leuchtend bei Nacht von dem Licht, das er schenkt, so umirrt sie die Erde. Immerfort blickt sie gebannt | hin auf den strahlenden Gott. ${ }^{130}$

Derrière ces vers il n'y a pas seulement, selon Popper, la raison pour laquelle la lumière de la lune n'est qu'un reflet du soleil ( $\dot{\alpha} \lambda \lambda \dot{o} \tau p$ pov $\phi \tilde{\omega} \varsigma)$, mais aussi une réflexion plus profonde sur les phases de la lune: certes, une

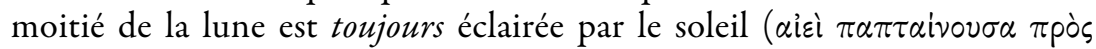

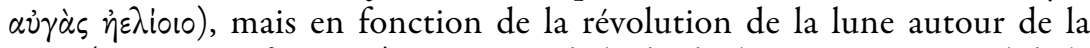

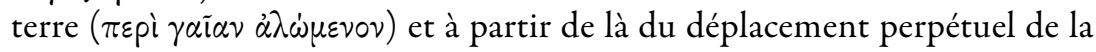
moitié qui est éclairée du point de vue de la terre, la partie de la lune visible-

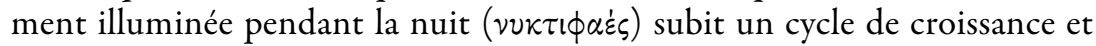
décroissance. Ce cycle, dans l'idée de Parménide, ne concerne cependant pas la lune elle-même, mais le changement perpétuel de la partie de sa moitié illuminée qui est visible par un terrien ${ }^{131}$. La lumière et notre vue ne sont pas fiables; ils nous font voir que la lune croît et décroît, alors qu'en réalité ni sa forme ni sa taille ne changent : elle reste la même, c'est-à-dire à l'origine une masse sphérique et obscure ${ }^{132}$.

En tout cas, c'est par une observation immanente et physique que la déesse montre en B 14-15, c'est-à-dire au cours de la doxa, le pouvoir

127. «How the Moon Might Throw Some of Her Light upon the Two Ways of Parmenides » («Comment la lune pourrait jeter un peu de sa lumière sur les deux voies de Parménide $\gg)$.

128. Le titre, le texte grec et la traduction en distiques élégiaques allemands se trouvent chez Popper 1992, p. 12 n. 1. Laissons de côté la question de savoir si les vers, en dépit du

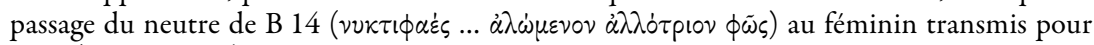
B $15(\pi \alpha \pi \tau \alpha i v o v \sigma \alpha)$, se suivaient vraiment : mais il est certain qu'ils vont ensemble par leur contenu.

129. « Déesse de la lune et dieu du Soleil ».

130. «Brillant pendant la nuit de la lueur d'un autre, / elle va vagabonde alentour de la terre, / Sans cesse se tournant vers le dieu rayonnant. »

131. Popper 1992, p. $14:$ : Mais selon la nouvelle découverte de Parménide, les phases de la lune [...] n'impliquaient pas de vrai changement ou mouvement de sa part. Il s'agit du résultat trompeur d'un jeu de lumière et d'ombre. »

132. Ibid.: « Nos sens nous induisent donc en erreur. Nous ne devons pas les croire. Ils nous trompent. Nous croyons que la lune bouge alors qu'en fait elle ne bouge pas. Mais c'est un jeu de lumière sur son corps obscur et immuable. » 
extrêmement trompeur de la lumière du soleil. La révélation de ce pouvoir est plus que toute autre apte à préparer à une critique générale des opinions qui reposent sur les sens, et par là à familiariser avec l'aletheia. À partir de là, la doxa ne fait pas que ramener les opinions des mortels à leurs deux principes, le Feu et la Nuit; mais ces principes eux-mêmes y sont examinés. À cela correspond la position intermédiaire, dans le voyage, du chemin des trajets du Jour et de la Nuit. Qui l'atteint par ses propres moyens est déjà « savant », loin au-dessus du monde quotidien des mortels; mais sur ce chemin, s'il reçoit l'aide d'une escorte divine, il peut aller encore plus loin : dans le domaine où il n'y a plus de jour. 


\section{BIBLIOGRAPHIE}

BARNeS, J. 1982 : The Presocratic Philosophers, Londres/New York, ${ }^{2} 1982$.

BECKER, O. 1937 : Das Bild des Weges und verwandte Vorstellungen im frühgriechischen Denken (= Hermes Einzelschriften, 4), Berlin, 1937.

Bernabé, A. 2004 : Poetae epici Graeci. Testimonia et fragmenta, II. Orphicorum et Orphicis similium testimonia et fragmenta, 1. Texte édité par -, Munich/Leipzig, 2004 (Bibliotheca scriptorum Graecorum et Romanorum Teubneriana).

- 2005 : Poetae epici Graeci. Testimonia et fragmenta, II. Orphicorum et Orphicis similium testimonia et fragmenta, 2. Texte édité par -, Munich/Leipzig, 2005 (Bibliotheca scriptorum Graecorum et Romanorum Teubneriana).

Bowra, C. M. 1937 : « The Proem of Parmenides », Classical Philology, 32 (1937), p. $97-$ 112.

Burkert, W. 1969 : « Das Proömium des Parmenides und die Katabasis des Pythagoras », Phronesis, 14 (1969), p. 1-30 (= Burkert 2008, p. 1-27).

- 2008: Kleine Schriften VIII - Philosophica (Th.A. Szlezák \& K.-H. Stanzel éd.), Göttingen, 2008 (Hypomnemata, Supplement, 2).

Campbell, D. A. 1991 : Greek Lyric III: Stesichorus, Ibycus, Simonides, and Others, texte édité et traduit, Cambridge MA, 1991 (The Loeb Classical Library).

Classen, C. J. 1965 : « Licht und Dunkel in der frühgriechischen Philosophie », Studium Generale 18 (1965), p. 97-116.

Coxon, A. H. 1986: The Fragments of Parmenides. A Critical Text with Introduction, Translation, the Ancient Testimonia \& a Commentary, Assen, 1986 (= Phronesis, Supplementary vol. 3).

DEICHGRÄBER, K. 1959 : « Parmenides’ Auffahrt zur Göttin des Rechts. Untersuchungen zum Prooimion seines Lehrgedichts », Akademie der Wissenschaften und der Literatur, Abhandlungen der Geistes- und Sozialwissenschaftlichen Klasse, 11 (1958), Wiesbaden.

Diels, H. 1897 : Parmenides Lehrgedicht, Berlin, 1897. [Réimpr. Sankt Augustin, 2003 (International Pre-Platonic Studies, 3), avec un avant-propos de W. Burkert.]

- 1912 : Die Fragmente der Vorsokratiker, $3^{\mathrm{c}}$ édition, Berlin, 1912.

Diels H. et W. KRANZ 1934 : Die Fragmente der Vorsokratiker, $5^{\mathrm{c}}$ édition, Berlin (6 édition augmentée par W. Kranz, Berlin, 1951).

FrÄNKEL, H. 1930: «Parmenidesstudien》, Nachrichten von der Gesellschaft der Wissenschaften zu Göttingen, philosophisch-historische Klasse, 1930, p. 153-192 (version modifiée dans Fränkel 1960, p. 157-197).

- 1960: Wege und Formen frühgriechischen Denkens. Literarische und philosophiegeschichtliche Studien, F. Tietze éd., Munich, 1960 [ $\left.{ }^{1} 1955\right]$.

- 1962: Dichtung und Philosophie des frühen Griechentums: eine Geschichte der griechischen Epik, Lyrik und Prosa bis zur Mitte des fünften Jahrbunderts, Munich, ${ }^{2} 1962$.

Furley, D. J. 1973 : « Notes on Parmenides », dans E. N. Lee, A. P. D. Mourelatos, R. M. Rorty (éd.), Exegesis and Argument. Studies in Greek Philosophy Presented to Gregory Vlastos, Assen, p. 1-15 (= Phronesis, Supplementary vol. 1).

GAllop, D. 1984 : Parmenides of Elea, Fragments. Text, Translation and Introduction, Toronto, 1984 (= Phoenix Supplementary, vol. 18 = Phoenix Pre-Socratics, 1).

Günther, H.-C. 1998 : Aletheia und Doxa: das Proömium des Gedichts des Parmenides, Berlin, 1998 (Philosophische Schriften, 27).

Heitsch, E. 1974 : Parmenides: Die Anfänge der Ontologie. Logik und Naturwissenschaft. Die Fragmente, texte édité, traduit et commenté, Munich, 1974 (TusculumBücherei). [ $2^{\mathrm{e}}$ édition révisée et augmentée, Munich, 1991.] 
HölSCher, U. 1969 : Parmenides : Vom Wesen des Seienden. Die Fragmente, griechisch und deutsch, texte édité, traduit et commenté, Francfort/Main, 1969 (Theorie, 1). $\left[{ }^{2} 1986.\right]$

KINGSLEY, P. 1999 : In the Dark Places of Wisdom, Inverness CA, 1999. [Réimpr. Londres, 2001.]

Kouremenos, T., G. M. Parássoglou et K. Tsantsanoglou 2006: The Derveni Papyrus, texte édité avec introduction et commentaire, Florence, 2006 (Studi e testi per il Corpus dei papiri filosofici greci e latini, 13).

KranZ, W. 1916: «Über Aufbau und Bedeutung des Parmenideischen Gedichtes », Sitzungsberichte der königlich-preussischen Akademie der Wissenschaften zu Berlin, 47 (1916), p. 1158-1176.

Mansfeld, J. 1964 : Die Offenbarung des Parmenides und die menschliche Welt, Assen, 1964 (Wijsgerige teksten en studies, 9).

- 2008: «A Crux in Parmenides Fr. B 1.3 DK », dans S. Heilen, R. Kirstein, R. Scott Smith, S. M. Trzaskoma, R. L. van der Wal, et M. Vorwerk (éd.), In Pursuit of "Wissenschaft". Festschrift für William M. Calder III zum 75. Geburtstag, Hildesheim/Zurich/New York, 2008 (Spudasmata, 119), p. 299-301.

Martin, A. et O. Primavesi 1999 : L'Empédocle de Strasbourg (P. Strasb. gr. Inv. 16651666), introduction, édition et commentaire, Berlin/New York, 1999.

Merlan, P. 1966 : « Neues Licht auf Parmenides », Archiv für Geschichte der Philosophie, 48 (1966), p. 267-276.

Morrison, J. S. 1955: «Parmenides and Er », Journal of Hellenic Studies, 75 (1955), p. 59-68.

MourelatoS, A. P. D. 1976 : « Determinacy and Indeterminacy, Being and Non-Being in the Fragments of Parmenides », dans R. Shiner \& J. King-Farlow (éd.), New Essays on Plato and the Pre-Socratics, Calgary, 1976 (= Canadian Journal of Philosophy, Supplementary vol. 2), p. 45-60.

Mutschmann, H. 1914 : Sexti Empirici Opera. II, Adversus dogmaticos libros V (Adv. Mathem. VII-XI) continens, Leipzig, 1914 (Bibliotheca scriptorum graecorum et romanorum Teubneriana).

Nussbaum, M. C. 1979: «Eleatic Conventionalism and Philolaus on the conditions of Thought », Harvard Studies in Classical Philology, 83 (1979), p. 63-108.

O’Brien, D. 1987: Le Poème de Parménide, texte, traduction, essai critique, en collaboration avec J. Frère pour la traduction française, (= Études sur Parménide, publiées sous la direction de P. Aubenque, tome I), Paris, 1987 (Bibliothèque d'histoire de la philosophie).

OWENS, J. 1979 : « Knowledge and "Katabasis" in Parmenides », Monist, 62 (1979), p. 1529.

Palmer, J. 2009 : Parmenides and Presocratic Philosophy, Oxford, 2009.

PellikaAn-Engel, M. E. 1974 : Hesiod and Parmenides. A new View on their Cosmologies and on Parmenides' Proem, Amsterdam, 1974.

Pfeiffer, H. 1975 : Die Stellung des Parmenideischen Lehrgedichtes in der epischen Tradition, Bonn, 1975 (Habelts Dissertationsdrucke, 21).

POPPER, K. R. 1992: « How the Moon Might Throw Some of Her Light upon the Two Ways of Parmenides », Classical Quarterly, 42 (1992), p. 12-19.

PRIMAVESI, O. 2005: «Theologische Allegorie: Zur philosophischen Funktion einer poetischen Form bei Parmenides und Empedokles », dans M. Horster \& C. Reitz (éd.), Wissensvermittlung in dichterischer Gestalt, Stuttgart, 2005 (Palingenesia, 85), p. 69-93.

- 2011: «Der Weg zur Offenbarung: Über Licht und Nacht im Proömium des Parmenides », dans G. Radke-Uhlmann \& A. Schmitt (éd.), Anschaulichkeit in 
Kunst und Literatur. Wege bildlicher Visualisierung in der europäischen Geschichte, Berlin/New York, 2011 (Colloquia Raurica, 11), p. 181-231.

Pugliese CarRatelli, G. 1963 : « Фं่خapXos », La parola del passato, 18 (1963), p. 385386.

RADT, S. 2005 : Strabons Geographika, vol. 4 : livres XIV-XVII, texte et traduction, Göttingen, 2005.

- 2009 : Strabons Geographika, vol. 8 : livres XIV-XVII, commentaire, Göttingen, 2009.

ReInHARDT, K. 1916: Parmenides und die Geschichte der griechischen Philosophie, Bonn, 1916 (Francfort/Main, ${ }^{4} 1985$ ).

SASSI, M. M. 1988 : « Parmenide al bivio. Per un'interpretazione del Proemio », La Parola del Passato, 43 (1988), p. 383-396.

Schmit, A. 2007 : « Parmenides und der Ursprung der Philosophie », dans E. Angehrn (éd.), Anfang und Ursprung. Die Frage nach dem Ersten in Philosophie und Kulturwissenschaft, Berlin/New York (Colloquia Raurica, 10), p. 109-139.

SEDLEY, D. 1999: « Parmenides and Melissus », dans A. A. Long (éd.), The Cambridge Companion to early Greek Philosophy, Cambridge, 1999 (The Cambridge Companions to Philosophy), p. 113-133.

STAHL, J. M. 1907 : Kritisch-historische Syntax des griechischen Verbums der klassischen Zeit, Heidelberg, 1907 (Indogermanische Bibliothek. 1. Abteilung. Sammlung indogermanischer Lehr- und Handbücher. 1. Reihe : Grammatiken. 4. Band).

STEuben, H. von 1981 : Parmenides, Über das Sein, griechisch-deutsch, mit einem einführ. Essay, Stuttgart, 1981 (Universal-Bibliothek, 7739).

- 1999: Wabrheit und Bekenntnis: Lichtoffenbarungen in antiker und christlicher Zeit, Möhnesee, 1999.

STEIN, H. 1867: «Die Fragmente des Parmenides ПEPI $\Phi \Upsilon \Sigma \mathrm{E} \Omega \Sigma »$, dans Symbola Philologorum Bonnensium in honorem Friderici Ritschelii collecta, II, Leipzig, 1867, p. 763-806.

TARÁn, L. 1965 : Parmenides: A Text with Translation, Commentary, and Critical Essays, Princeton, NJ, 1965.

VOS, H. 1963 : « Die Bahnen von Nacht und Tag », Mnemosyne, 16 (1963), p. 18-34.

WeST, M. L. (éd.) 1966: Hesiod, Theogony, ed. with prolegomena and comm., Oxford, 1966.

- (éd.) 1972: Iambi et Elegi Graeci ante Alexandrum cantati. II, Callinus, Mimnermus. Semonides, Solon, Tyrtaeus, Minora adespota, Oxford, 1972 ( $2^{\mathrm{c}}$ éd. augmentée et corrigée, Oxford, 1992).

Wiesner, J. 1996 : Parmenides, Der Beginn der Aletheia. Untersuchungen zu B 2 - B 3 - B 6, Berlin/New York, 1996.

WilamowITZ U. von 1899: «Lesefrüchte (XXIV-XXXVIII)», Hermes, 34 (1899), p. 203-230 (= U. von Wilamowitz-Moellendorff, Kleine Schriften. IV, Lesefrüchte und Verwandtes, Berlin, 1962, p. 45-71).

- 1902: Griechisches Lesebuch, Bd. I (Text), 1. Halbband, Berlin, 1902.

- 1931 : Der Glaube der Hellenen, Bd. I, Berlin, 1931. 